\title{
Efficacy of platelet-rich fibrin in promoting the healing of extraction sockets: a systematic review
}

Sarah Al-Maawi ${ }^{1}$, Kathrin Becker ${ }^{2}$, Frank Schwarz ${ }^{3}$, Robert Sader ${ }^{1}$ and Shahram Ghanaati ${ }^{*}$

\begin{abstract}
Purpose: To address the focused question: in patients with freshly extracted teeth, what is the efficacy of platelet-rich fibrin (PRF) in the prevention of pain and the regeneration of soft tissue and bone compared to the respective control without PRF treatment?

Methods: After an electronic data search in PubMed database, the Web of Knowledge of Thomson Reuters and hand search in the relevant journals, a total of 20 randomized and/or controlled studies were included.

Results: $66.6 \%$ of the studies showed that PRF significantly reduced the postoperative pain, especially in the first 1-3 days after tooth extraction. Soft tissue healing was significantly improved in the group of PRF compared to the spontaneous wound healing after 1 week (75\% of the evaluated studies). Dimensional bone loss was significantly lower in the PRF group compared to the spontaneous wound healing after 8-15 weeks but not after 6 months. Socket fill was in $85 \%$ of the studies significantly higher in the PRF group compared to the spontaneous wound healing.

Conclusions: Based on the analyzed studies, PRF is most effective in the early healing period of 2-3 months after tooth extraction. A longer healing period may not provide any benefits. The currently available data do not allow any statement regarding the long-term implant success in sockets treated with PRF or its combination with biomaterials. Due to the heterogeneity of the evaluated data no meta-analysis was performed.
\end{abstract}

Keywords: PRF, Platelet-rich fibrin, Socket preservation, Ridge preservation, Socket healing, Pain management, Soft tissue healing

\section{Introduction}

Dental implants have become an integral part of the oral and maxillofacial surgery. They provide the most comfortable and favorable method to replace lost teeth and reconstruct the esthetic and function for the patients [1, 2]. To achieve long-term success of dental implants many clinical, biomechanical and biological requirements are needed $[3,4]$. Especially, healthy and active bone and soft

\footnotetext{
*Correspondence: Shahram.Ghanaati@kgu.de

' FORM, Frankfurt Oral Regenerative Medicine, Clinic for Maxillofacial and Plastic Surgery, Goethe University, Theodor-Stern-Kai 7, 60596 Frankfurt/ Main, Germany

Full list of author information is available at the end of the article
}

tissue are needed to support osseointegration. Therefore, a thorough understanding of the mechanisms of socket healing became a central research topic in the last decades [5, 6]. After tooth loss, the alveolar bone undergoes a remodeling process resulting in loss of bone quantity and changes of bone quality [7]. These processes finally lead to alveolar bone atrophy. The process of atrophy was described as a rapid and continuous process. In this context, $50-60 \%$ of the alveolar bone atrophies in the first three months after tooth extraction $[6,8]$. These findings highlight the importance of the initial period after tooth extraction as critical for the further healing and changing of the alveolar bone. Accordingly, different protocols 
were established to avoid bone atrophy and achieve dental implantation.

Socket preservation is a prophylactic intervention that includes applying bone substitute materials (BSMs) into the extraction socket to preserve the alveolar bone dimension $[9,10]$. Similarly ridge preservation is applied when tooth extraction results in a larger defect. A wide range of BSMs including synthetic and naturally derived biomaterials is available for clinical application [11, 12]. After BSMs application, a healing period of 3-6 months is recommended according to the defect morphology and the applied BSM [13-15]. During the healing period of 3-6 months, the processes of natural alveolar healing interferes with the BSM-based new bone formation and leads to the regeneration of a sufficient implant bed, that allows the delayed insertion of dental implant [16]. This two-stage implantation concept is based on the preparation of the alveolar bone prior to implant insertion. Many clinical studies reported about socket and ridge preservation using different types of BSMs [17]. However, there is still no clear evidence about the most suitable time of implant placement [18] Immediate implant placement after tooth extraction has been considered an alternative option to limit alveolar bone resorption [18, 19]. However, this approach is limited to specific socket morphologies and indications, when a sufficient bone volume is available and the buccal bone is preserved. This method can be also applied in combination with BSMs to fill the socket when needed [20].

In addition to BSMs, blood concentrate systems gained increasing importance in different fields of regenerative medicine in the last decade [21]. Blood concentrates are obtained from patients own peripheral blood [22]. Thereby, the blood components such as leukocytes, platelets, plasma proteins and growth factors are concentrated by centrifugation and prepared using different protocols [23-25]. Platelet-rich plasma (PRP) is the first generation of blood concentrates. PRP includes mainly platelets, whereas leukocytes are removed during the preparation process $[25,26]$. For its preparation, the patients' blood is centrifuged in two centrifugation steps [27]. In addition, plasma rich in growth factors (PRGF) is a further concept that utilizes the advantages of blood-derived growth factors [28]. Both systems apply a rather high relative centrifugal force (RCF) during their preparation [25]. By contrast, the second generation of blood concentrates, i.e., platelet-rich fibrin (PRF), is prepared by a one-step centrifugation without the application of any anticoagulants [29]. PRF consists of platelets, leukocytes and their subgroups embedded in a fibrin matrix with plasma proteins [21]. The first protocol of PRF applies a comparably lower, but still high RCF $(\approx 710 \times g)$ [30]. This protocol was called leukocytes-rich platelet-rich fibrin (L-PRF), mainly because it contains more leukocytes compared to the first-generation blood concentrates PRP.

PRF matrices have been used in different indications in oral and maxillofacial surgery and implant dentistry [31]. Some clinical studies reported on the benefits and drawbacks of the different blood concentrate systems [32]. Recently, different systematic reviews aimed to summarize the available evidence on the use of PRF [31, 33, 34]. However, they were not focused on socket preservation, but extended their investigation to a wider range of indications and included different evidence levels. Therefore, the present systematic review aimed to focus on the role of PRF in ridge preservation to addressed the following focused questions: in patients with freshly extracted teeth, what is the efficacy of PRF in the prevention of pain and the regeneration of soft tissue and bone compared to the respective control without PRF treatment?

\section{Methods}

This systematic review was designed and performed following the preferred reporting items of the PRISMA statement $[35,36]$.

\section{Focused question}

This systematic review followed the structure of the focused questions (PICO) for the literature search [37]:

- Population (P): patients with freshly extracted teeth.

- Intervention (I): socket or ridge preservation using platelet-rich fibrin (PRF) with or without biomaterials.

- Comparison (C): spontaneous healing, biomaterials without PRF.

- Outcomes (O): measurements of at least one of the following parameters: postoperative inflammation and pain, soft tissue healing, dimensional bone volume changes, bone quality.

\section{Search strategy}

An electronic search was conducted through PubMed and Web of Science, followed by a hand search for relevant articles published between 1990 up to June 2021. A commercially available software program (Microsoft Excel) was used for data management. Two authors (S.A. and S.G.) independently screened the identified articles. In case of disagreement regarding inclusion, detailed review of the defined criteria was performed and the disagreements were resolved upon discussion.

The combination of following keywords:

"PRF", "platelet rich fibrin", "socket preservation", "ridge preservation", "molar", "premolar". 
Keywords combination:

("platelet rich fibrin"[MeSH Terms] OR ("plateletrich"[All Fields] AND "fibrin"[All Fields]) OR "platelet-rich fibrin"[All Fields] OR "PRF"[All Fields] OR ("platelet"[All Fields] AND "rich"[All Fields] AND "fibrin"[All Fields]) OR "platelet rich fibrin"[All Fields]) AND ("socket" or "ridge" or "molar" or "premolar"[All Fields]).

A manual search was additionally performed in the following journals:

- International Journal of Oral and Maxillofacial Implants;

- Clinical Implant Dentistry and Related Research;

- Clinical Oral Implants Research;

- Journal of Clinical Oral Investigations

- Journal of Implantology;

- Journal of Oral and Maxillofacial Surgery;

- International journal of oral and maxillofacial surgery.

\section{Inclusion criteria}

- English language

- Patient age 15-99 years

- Prospective controlled (CCTs) and/or randomized clinical studies (RCTs) in humans with either a splitmouth or parallel design with reasonable controls*

- Treatment of fresh sockets/ridge

- Treatment using either PRF (with or without biomaterials, i.e., bone substitute materials, collagen membranes as well as any other membrane of different origin) or spontaneous healing

- Treatment without any additional chemical or physical agents in/on the alveolus after extraction except suture materials

- Subject with and without anticoagulation intake.
- Immediate implantation;

- Inadequate methods or reporting of the study design and/or patients' data.

"reasonable controls were considered as control groups in which all applied procedures were equivalent to the test group except for PRF. Therefore, in case of the sole use of PRF in the test group, the reasonable control was considered as the spontaneous healing. In the case of the use of biomaterials in combination with PRF in the test group, the reasonable control was considered to be the application of the exact same biomaterial without PRF.

\section{Quality evaluation of included studies}

The quality of selected RCTs was reviewed to assess the bias risk. Evaluation was performed according to the Cochrane Handbook for Systematic Reviews of Interventions version 6.2 (updated February 2021), (low, high, unclear). CCTs were evaluated according to NewcastleOttawa Quality Assessment Scale for non-randomized studies. The following categories were analyzed: random sequence generation, allocation concealment, blinding of participants and personnel, blinding of outcome assessment, and incomplete outcome data [38]. The assessment was conducted by two independent reviewers (SA, SG) based on the published full text article. Disagreements were resolved upon discussion.

\section{Data extraction}

Data extraction was organized in a data-sheet including, study design, number of treated subjects, case definition, population, surgical extraction protocol, socket specifications, PRF-preparation protocol, treated groups comparison. For data analysis following parameters were defined:

- Primary outcomes: radiological and clinical evaluation of bone regeneration, dimensional bone-level change and histological assessment of bone regeneration.

- Secondary outcomes: healing period, pain management outcome and soft tissue regeneration.

\section{Results}

Study inclusion

The PubMed and Web of Science search resulted in 312 and 215, respectively. The manual search in the relevant journals did not result in additional titles. One article was retrieved from other sources (published reviews). After removal of 215 duplicated articles, 312 titles and abstracts were reviewed from which 292 studies were excluded according to the exclusion criteria. Thirtythree full-text articles were reviewed, of which 20 were 


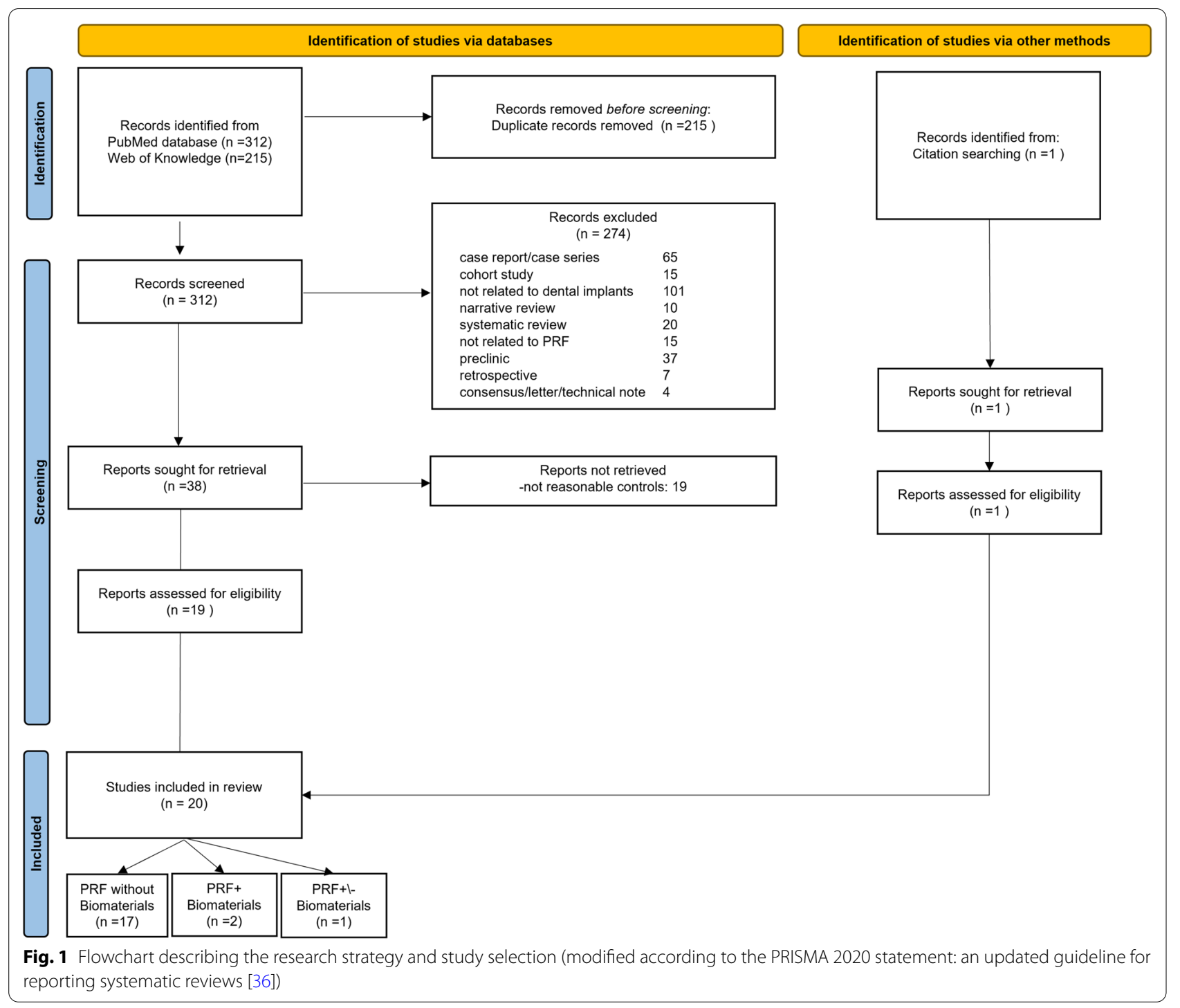

included in the qualitative analysis. Due to parameter variation and data limitation, no meta-analysis could be conducted (Fig. 1).

\section{Study designs}

Twenty studies were analyzed in this review. Nine of the included studies were designed as parallel RCTs and seven were designed as split mouth RCT. Further two CCTs (one split mouth and one parallel study) were included in this review. One study was not further defined by the authors and one further study included both split mouth and parallel design according to the teeth needed to extract in each patient. Seventeen studies compared only the treatment of PRF as a test group to the spontaneous healing without any further treatment. One study included four groups and evaluated first the treatment of PRF alone in comparison to the spontaneous wound healing and second the combination of PRF with a bone substitute material in comparison to the bone substitute material alone [39]. Two further studies compared Bone substitute materials in combination with PRF to Bone substitute materials without PRF.

The case definition differed in the respective studies. Mainly patients in need of tooth extraction with or without dental implantation were studied. Additionally, some studies focused on single rooted teeth or premolars only. Most of the studies did not report or specify the morphology of the treated sockets/ridge. When reported, the studies included sockets with presence of $50 \%$ or more of the lingual/buccal socket walls. Most of the studies reported atraumatic tooth extraction without flap mobilization or intention of primary healing (Table 1). 


\section{Evaluation of bias risk}

The reviewer judgment on the bias risk of RCTs showed that the highest bias risk was assessed in the categories blinding of participant and personnel as well as blinding of outcome assessment (Table 2, Fig. 2). The highest bias risk of the two included CCTs was referred to case selection and comparability (Table 3).

\section{PRF preparation protocol}

Most of the included studies evaluated the L-PRF protocol. Additionally, Giudice et al. [40] and Castro et al. [41] evaluated A-PRF + in comparison to L-PRF, Clark et al. [39] evaluated A-PRF and Ustaoglu et al. [42] analyzed T-PRF in comparison to L-PRF. Most of the studies reported solely the used rounds per minutes (rpm) and centrifugation time without referring to the centrifuge design or the applied relative centrifugal force (Table 4).

\section{Primary outcomes}

The results of the primary outcomes are described below.

\section{Bone regeneration}

Fifteen of 20 studies evaluated the dimensional bonelevel changes and bone regeneration using different methods (Table 5).

Clinical evaluation. Kumar et al. [43] applied a clinical measurement method using metal capillaries to assess the width and height bone loss after 6 months. In both cases, no statistical significant differences were found between the PRF and the control group (spontaneous wound healing). Additionally, Clark et al. [39] evaluated the bone dimension change after an average of 15 weeks (3.75 months) using alginate impression and periodontal probe. The results showed that the A-PRF group underwent significantly lower ridge height reduction compared to the control group. However, no statistical significant differences were found when assessing the alveolar bone width. Alzahrani et al. [44] analyzed the alveolar ridge with reduction after 1,4 and 8 weeks using cast analysis. The results showed statistically significantly lower reduction in the PRF group after 4 and 8 weeks ( 1 and 2 months) compared to the control group. Moreover, Suttapreyasri et al. [45] did not show any statistical significant difference in the alveolar bone width and buccal and lingual contour changes using cast analysis after 8 weeks. Hauser et al. [46] reported on statistically significantly lower percent of alveolar crest width resorption in the PRF group compared to the control group after 8 weeks (Table 5).

Cone beam computer tomography. (CBCT) CBCT measurements of the dimensional bone alteration after 3 months were performed by Canellas et al. [47] the results showed statistically significantly lower bone resorption in the PRF group compared to the control group especially in the $1-3 \mathrm{~mm}$ below the alveolar crest and the buccal wall. However, no difference was shown, when evaluating the horizontal bone loss. Additionally, the total volume of new bone formation was statistically significantly higher in the PRF group compared to the control group. Similarly, Srinivas et al. [48] showed statistically significantly higher bone density in the PRF group after 3 months by CBCT analysis. However, no differences were found in the bone height change. A further study by Zhang et al. [49] performed CBCT analysis to assess bone resorption after 3 months. They showed markedly lower resorption in all dimensions in the PRF group compared to the control group. However, they did not report statistical significant differences. Moreover, Temmerman et al. [50] analyzed the bone changes using $\mathrm{CBCT}$ and showed significantly lower vertical resorption in the PRF group compared to the control group, especially in the buccal wall. Similarly, a significantly lower horizontal bone resorption was shown in the $1-5 \mathrm{~mm}$ below the alveolar crest in the PRF group compared to the control group. The percent socket fill was significantly higher in the PRF group compared to the control group. Castro et al. [41] also demonstrated a significantly higher socket fill after 3 months using CBCT measurement in the PRF treated group compared to the untreated control (Table 5).

Clark et al. [39] also analyzed the dimensional ridge reduction after treatment using a bone substitute material in combination with PRF compared the treatment using the biomaterial alone. No statistical significant differences were shown in the clinical evaluation. Two further studies analyzed the socket augmentation using PRF in combination with bone substitute materials compared to the augmentation using native bone substitute material without PRF. Thakkar et al. [52] showed that the addition of PRF significantly reduce the ridge width reduction after 6 months. However, no significant difference was found when evaluating the ridge height reduction. Yewale et al. [51] showed significantly higher alveolar width preservation in the group of A-PRF + only when measured at $3 \mathrm{~mm}$ below the alveolar crest (Table 5 ).

Two-dimensional X-ray evaluation. Conventional radiologic analysis performed by Ahmed et al. [53] showed significantly lower resorption in the PRF group after 4 months compared to the control group. Kumar et al. [43] showed no statistical significant differences between the groups when considering the percent of socket fill after 6 months. By contrast Alzahrani et al. [44] used similar evaluation method and showed significantly higher percent of bone fill in the PRF group after 2 months compared to the control group. Suttapreyasri et al. [45] analyzed the resorption of marginal bone at the 


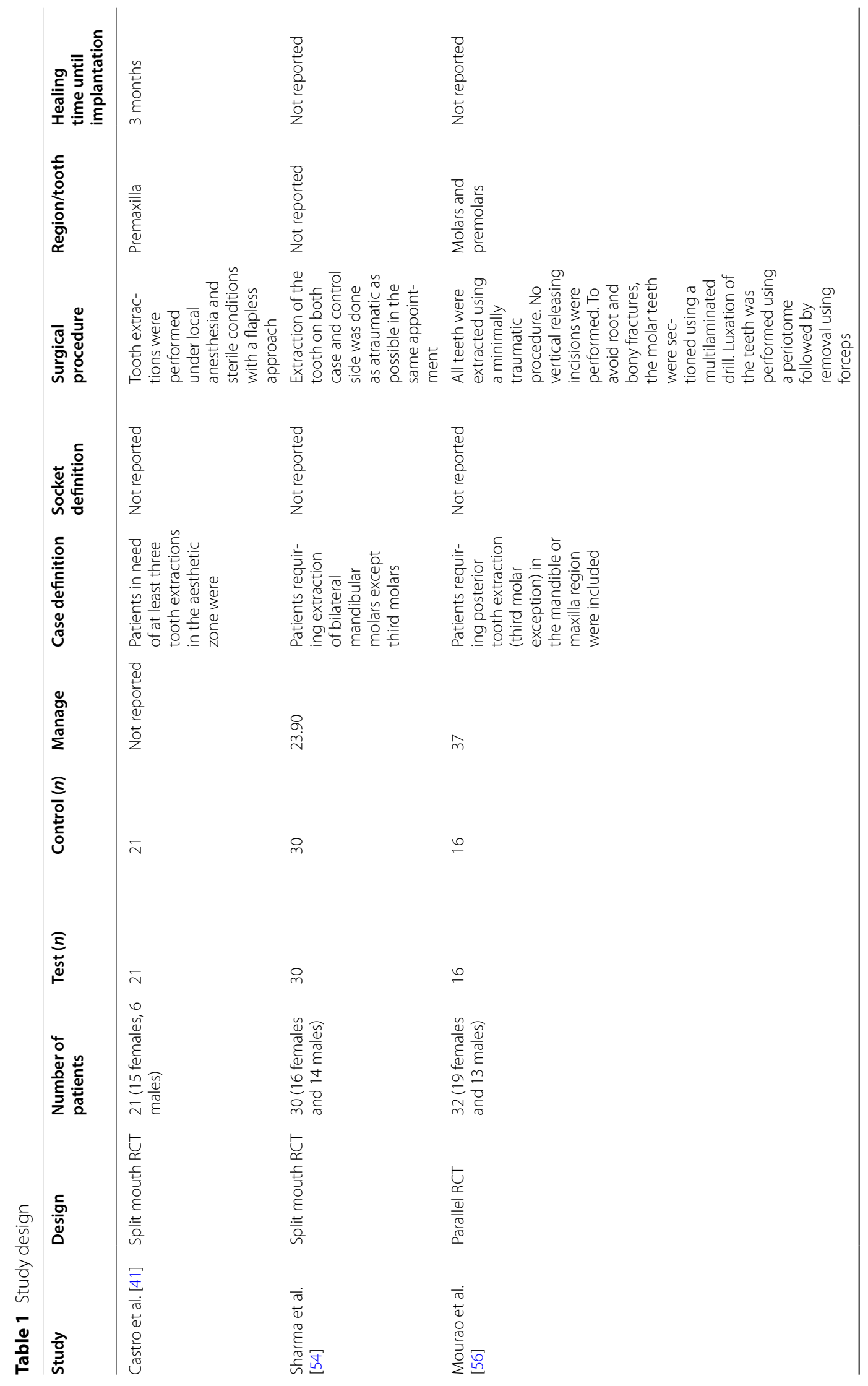




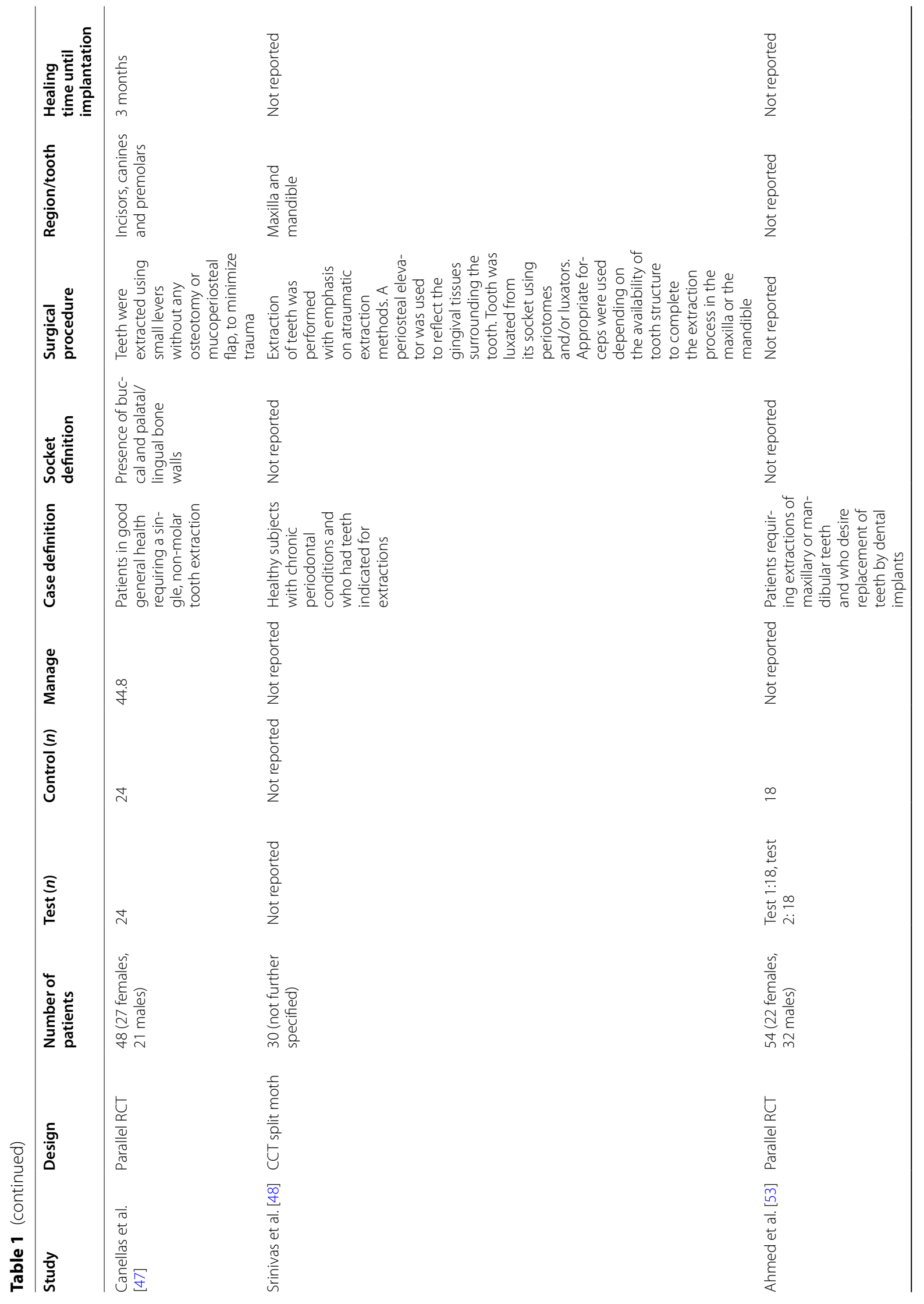




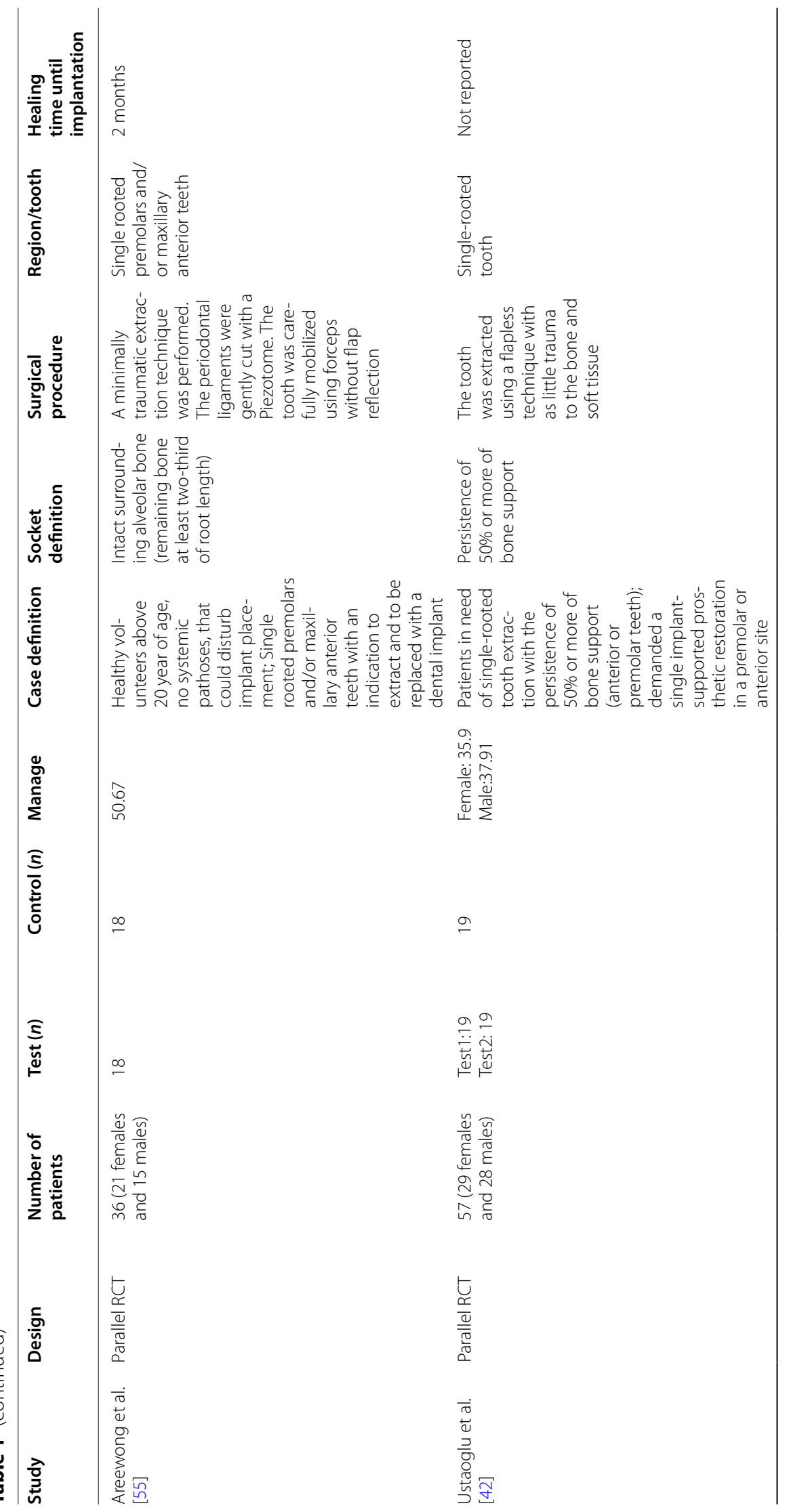




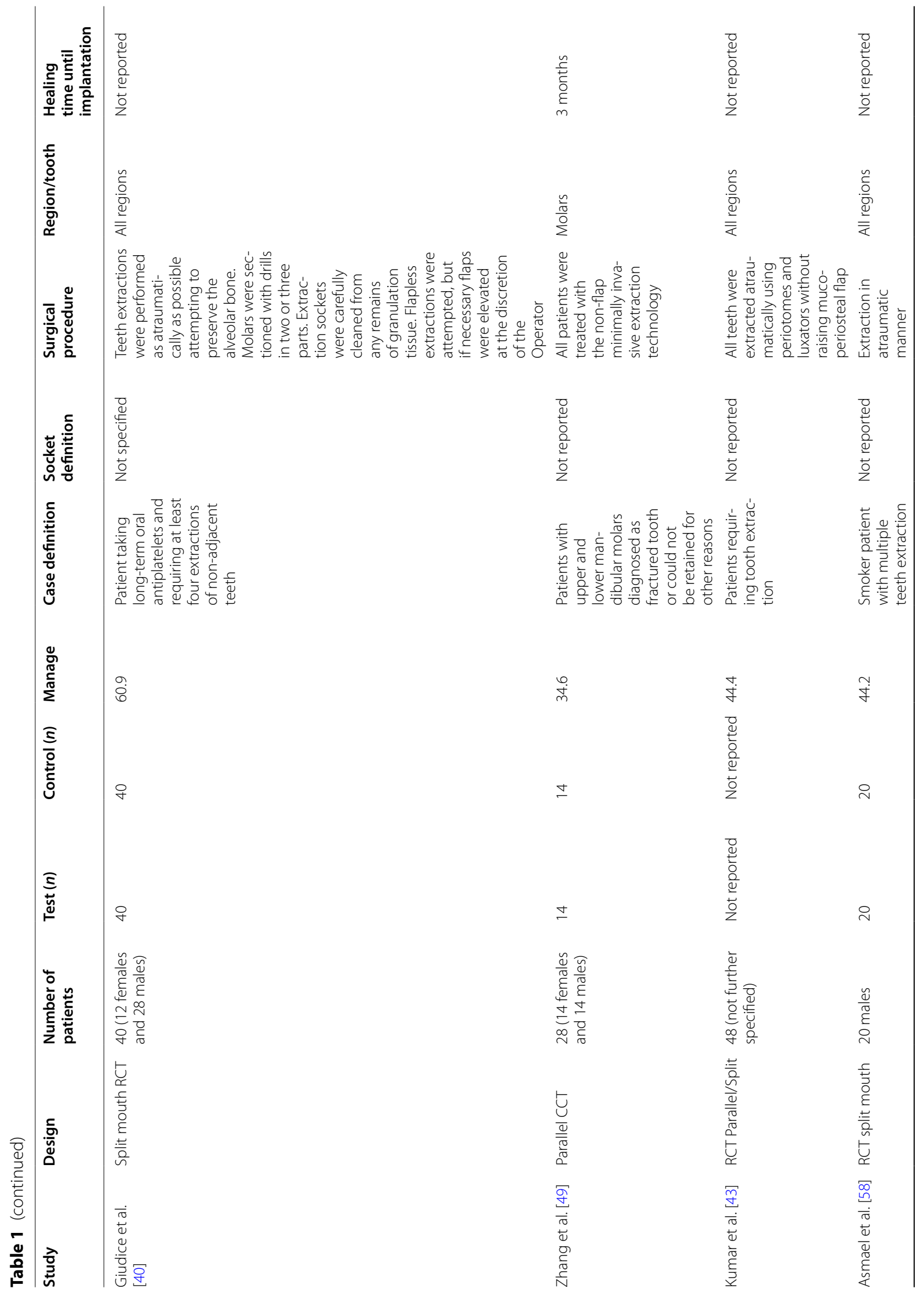




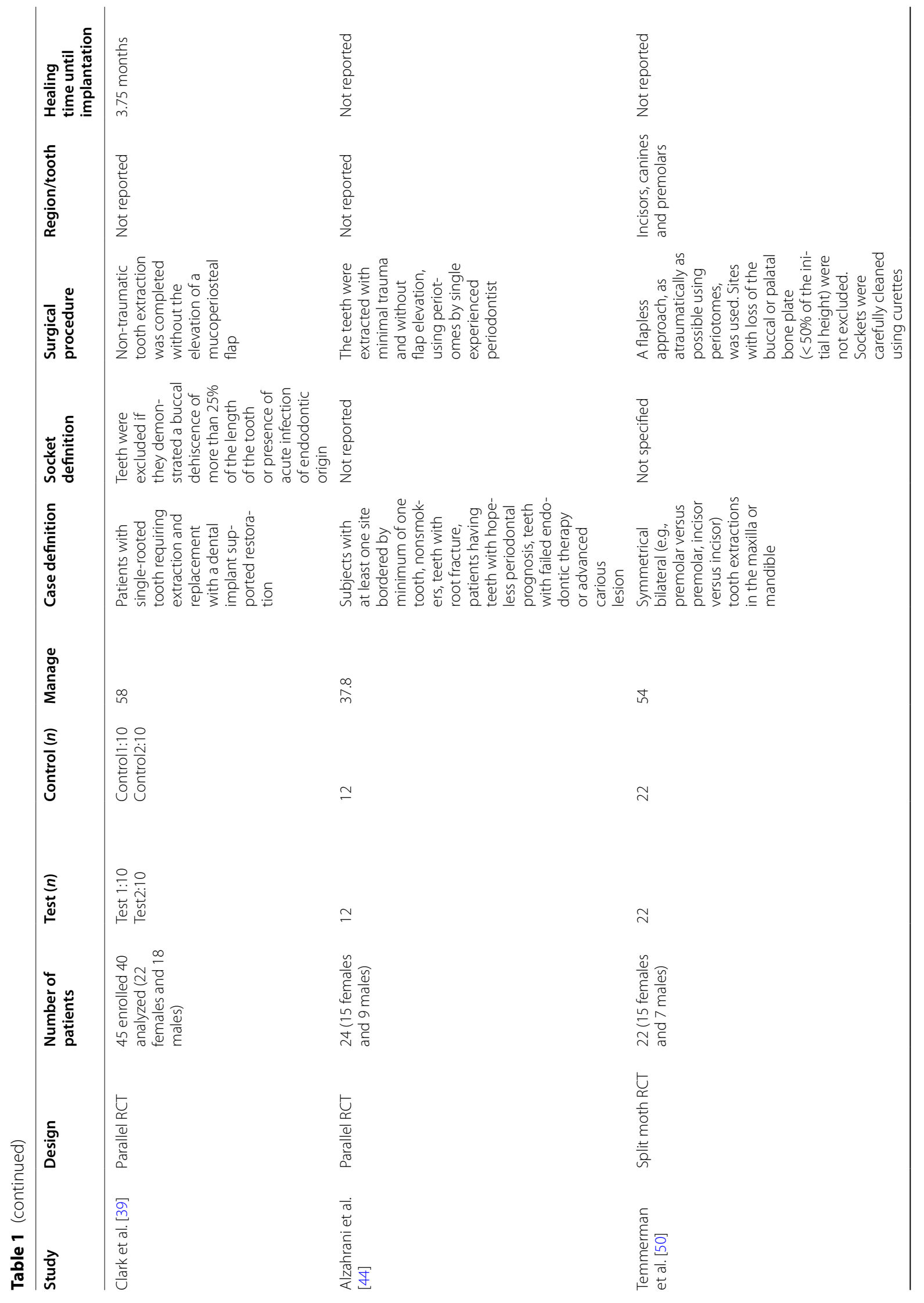




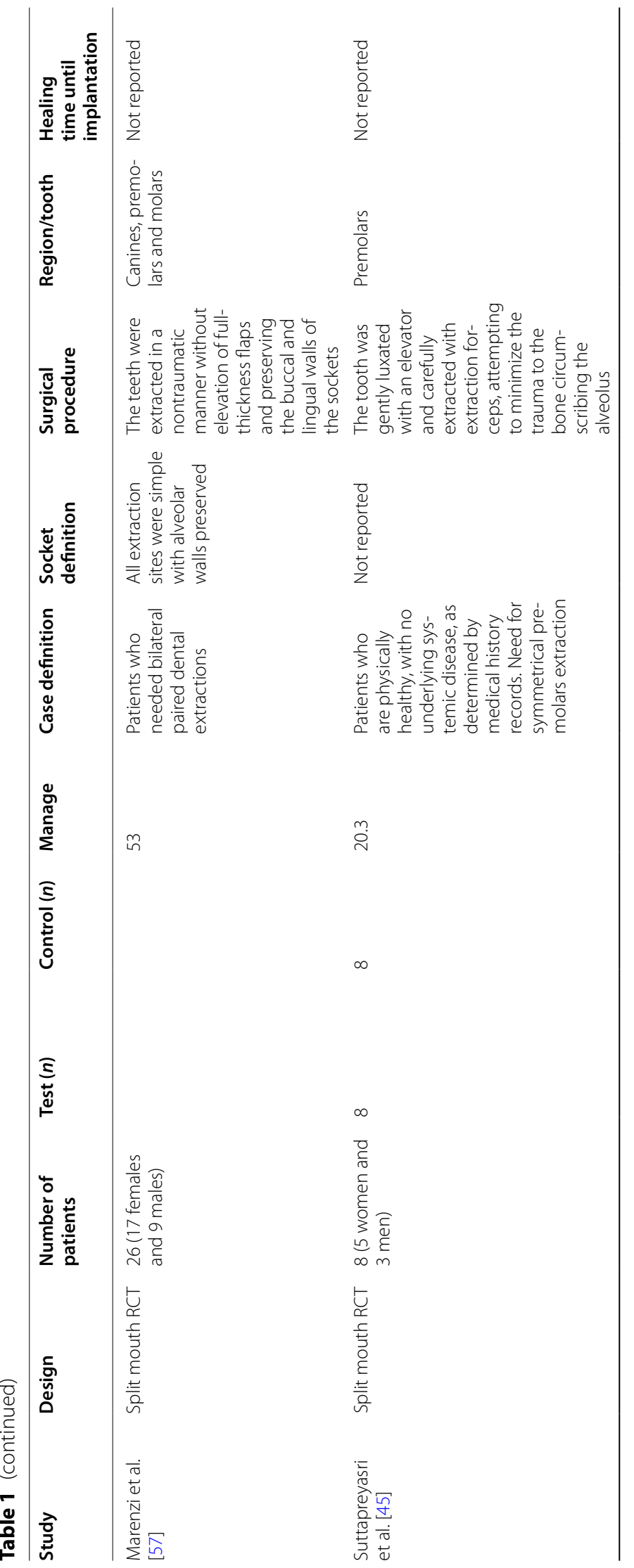




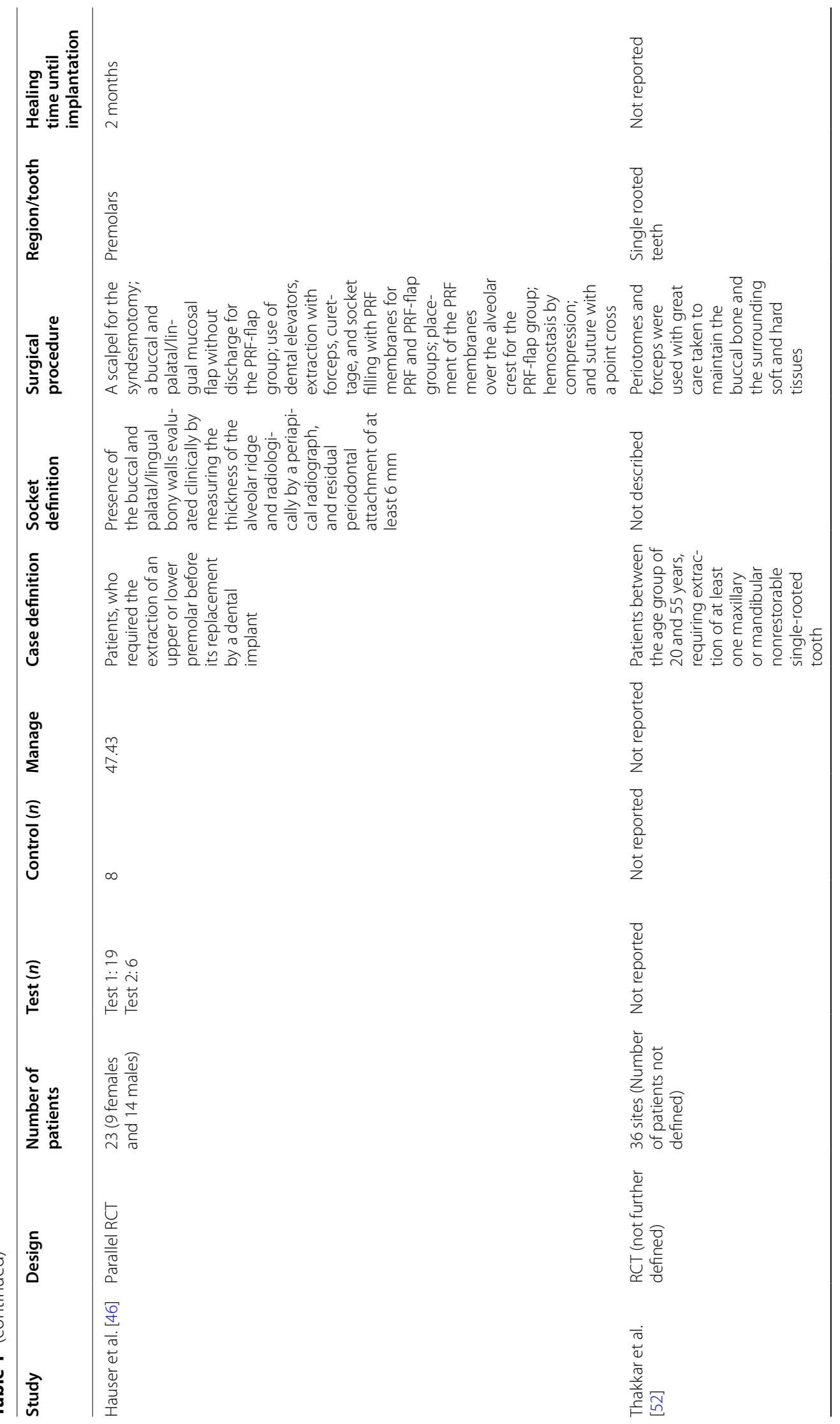




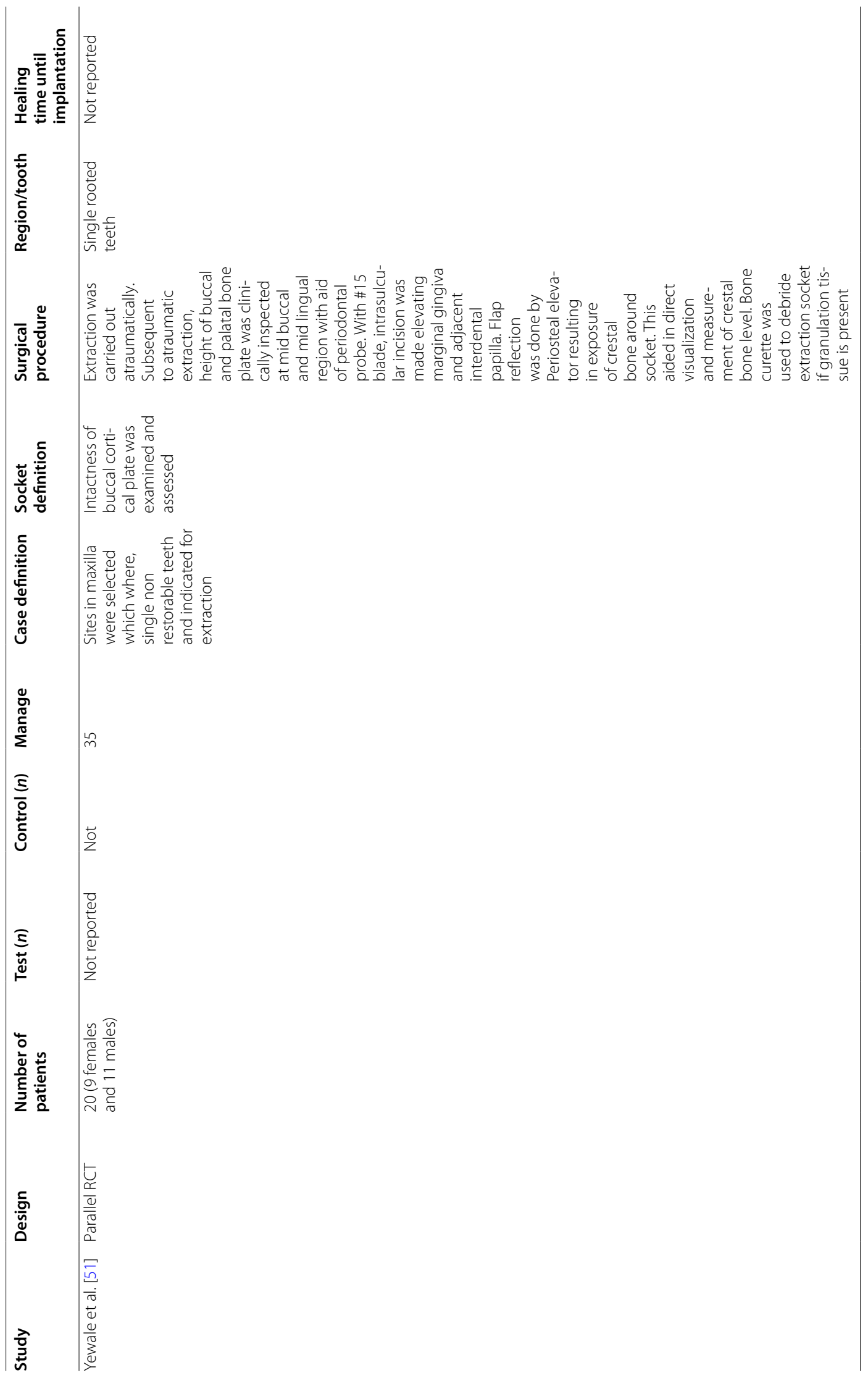


Table 2 Risk bias assessment according to according to the Cochrane collaborations tool

\begin{tabular}{|c|c|c|c|c|c|}
\hline Study & $\begin{array}{l}\text { Random sequence } \\
\text { generation }\end{array}$ & $\begin{array}{l}\text { Allocation } \\
\text { concealment }\end{array}$ & $\begin{array}{l}\text { Blinding of participants } \\
\text { and personnel }\end{array}$ & $\begin{array}{l}\text { Blinding of outcome } \\
\text { assessment }\end{array}$ & $\begin{array}{l}\text { Incomplete } \\
\text { outcome } \\
\text { data }\end{array}$ \\
\hline Castro et al. [41] & + & n.a & - & + & + \\
\hline Sharma et al. [54] & + & n.a & - & - & + \\
\hline Mourao et al. [56] & + & n.a & - & - & + \\
\hline Canellas et al. [47] & + & n.a & + & + & + \\
\hline Ahmed et al. [53] & - & n.a & - & - & + \\
\hline Areewong et al. [55] & + & n.a & - & - & + \\
\hline Ustaoglu et al. [42] & + & n.a & $\begin{array}{l}\text { Participant - } \\
\text { Personnel + }\end{array}$ & + & + \\
\hline Giudice et al. [40] & + & n.a & - & + & + \\
\hline Kumar et al. [43] & - & n.a & $?$ & - & + \\
\hline Asmael et al. [58] & - & n.a & - & - & - \\
\hline Clark et al. [39] & + & n.a & $?$ & + & + \\
\hline Alzahrani et al. [44] & - & n.a & - & - & + \\
\hline Temmerman al. [50] & + & n.a & - & - & + \\
\hline Marenzi et al. [57] & + & n.a & $?$ & $?$ & + \\
\hline Suttapreyasri et al. [45] & - & n.a & - & - & + \\
\hline Hauser et al. [46] & $?$ & n.a & - & $?$ & + \\
\hline Thakkar et al. [52] & + & n.a & - & - & + \\
\hline Yewale et al. [51] & + & n.a & - & + & + \\
\hline
\end{tabular}

+ low ristk, -high risk, ? unclear risk, n.a., not applicable

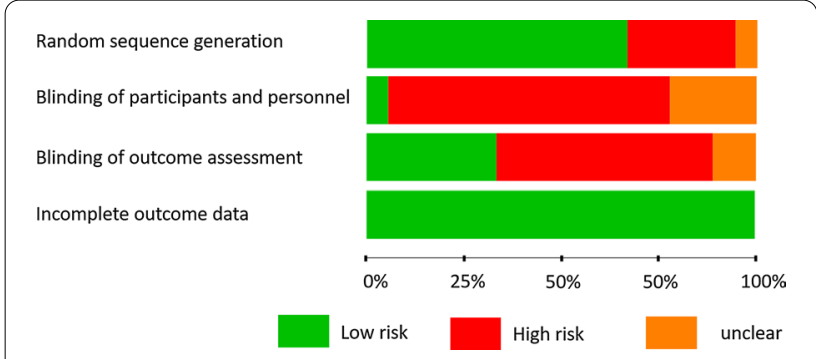

Fig. 2 Bias risk assessment of RCTs according to the Cochrane collaborations tool

Table 3 Risk bias assessment according to Newcastle-Ottawa Quality Assessment Scale case-control studies

\begin{tabular}{|c|c|c|c|}
\hline & Selection & Comparability & Exposure \\
\hline Srinivas et al. [48] & 支 & 衣 & 衣文 \\
\hline Zhang et al. [49] & 衣公 & 文 & 文文 \\
\hline
\end{tabular}

mesial and distal sites after 2 months and did not show statistically significant differences. Whereas Hauser et al. [46] performed similar measurements after 2 months and showed statistically significant differences, especially in the mesial site. Moreover, Sharma et al. [54] did not show statistical significant differences, when analyzing the bone density by means of gray scale after 16 weeks of healing (Table 5).

Histologic evaluation. Four of the 20 evaluated studies analyzed bone core biopsies by histology. Focus was placed on the evaluation of the percent of new bone formation by histomorphometry. Canellas et al. [47]; Zhang et al. [49] and Castro et al. [41] showed significantly higher percent of new bone formation in the PRF group after 3 months compared to the control group. Areewong et al. [55] (healing time: 8 weeks) and Clark et al. [39] (healing time 15 weeks) did not show statistical significant differences in the ratio of new bone formation (Table 5).

Micro-computer tomography (micro-CT) Clark et al. [39] and Hauser et al. [46] analyzed core biopsies using micro-CT. Bone volume to tissue volume analysis after 8 weeks did not show any differences between the PRF and control group. Similarly, the bone density measurement after 15 weeks did not show statistically significant differences. Castro et al. [41] showed a statistically significantly higher percent of bone volume/ tissue volume when comparing the group of A-PRF + to the untreated control. However, no statistical significant differences were documented for the L-PRF group (Table 5). 
Table 4 Centrifugation protocols used in each study

\begin{tabular}{|c|c|c|c|c|c|}
\hline Study & PRF-type & Tube & $\operatorname{RPM}(\operatorname{RCF}[\times \mathbf{g}])$ & $\begin{array}{l}\text { Centrifugation } \\
\text { time (min) }\end{array}$ & Centrifuge \\
\hline \multirow[t]{2}{*}{ Castro et al. [41] } & L-PRF & $\begin{array}{l}\text { 9ml silica-coated plastic tubes } \\
\text { without anticoagulant (BVBCTP- } \\
\text { 2, Intra-Spin, Intra-Lock) }\end{array}$ & $2700 \mathrm{rpm}\left(\mathrm{RCF}_{\text {clot }}: 408 \mathrm{~g}\right)$ & 12 & Intra-Spin, Intra-Lock \\
\hline & A-PRF + & $\begin{array}{l}\text { 10-ml glass tubes without anti- } \\
\text { coagulant (DUO) for A-PRF }+\end{array}$ & $1300 \mathrm{rpm}\left(\mathrm{RCF}_{\text {clot: }}: 145 \mathrm{~g}\right)$ & 8 & DUO Process \\
\hline Sharma et al. [54] & PRF & $\begin{array}{l}6 \mathrm{ml} \text { intravenous blood was } \\
\text { collected in a 10-ml sterile tube } \\
\text { without anticoagulant }\end{array}$ & 3000 rpm & 10 & $\begin{array}{l}\text { LabTech AVI-532-BL centrifugation } \\
\text { machine }\end{array}$ \\
\hline Mourao et al. [56] & L-PRF & $\begin{array}{l}\text { 10-ml red tubes (IntraSpin }{ }^{\mathrm{TM}}, \\
\text { Biohorizons }^{\circledR} \text { ) }\end{array}$ & 2700 rpm $(708 \times 9)$ & 12 & $\begin{array}{l}\text { IntraSpin }{ }^{\mathrm{TM}} \text {, Biohorizons }{ }^{\circledR}, \text { Birming- } \\
\text { ham, Alabama, USA }\end{array}$ \\
\hline Canellas et al. [47] & L-PRF & sterile, glass-coated plastic tubes & $2700(708 \times 9)$ & 12 & $\begin{array}{l}\text { Intra-Lock, Boca Raton, Florida, } \\
\text { USA }\end{array}$ \\
\hline Srinivas et al. [48] & L-PRF & $\begin{array}{l}10 \mathrm{ml} \text { test tubes which were kept } \\
\text { without an anticoagulant }\end{array}$ & 3000 rpm & 10 & Not reported \\
\hline Ahmed et al. [53] & L-PRF & Not reported & 3000 rpm & 10 & Not reported \\
\hline Areewong et al. [55] & L-PRF & Glass tube & 2700 rpm & 12 & IntraSpin, Intra-Lock, Nice, France \\
\hline \multirow[t]{2}{*}{ Ustaoglu et al. [42] } & L-PRF & $9 \mathrm{~mL}$ tubes & 2700 rpm & 12 & $\begin{array}{l}\text { Intra-Spin System, L-PRF kit, Intra- } \\
\text { Lock, Boca-Raton, FL, USA }\end{array}$ \\
\hline & T-PRF & Grade IV sterile titanium tubes & 2800 rpm & 12 & Not reported \\
\hline \multirow[t]{2}{*}{ Giudice et al. [40] } & A-PRF + & A-PRF + tubes & 1300 rpm & 8 & $\begin{array}{l}\text { DUO centrifuge (Process for PRF, } \\
\text { Nice, France); }\end{array}$ \\
\hline & L-PRF & Red tubes & 2700 rpm & 18 & $\begin{array}{l}\text { (Intra-Lock International, Boca } \\
\text { Raton, Florida, USA }\end{array}$ \\
\hline Zhang et al. [49] & L-PRF & $\begin{array}{l}\text { test tubes without any antico- } \\
\text { agulant }\end{array}$ & $400 \times 9$ & 10 & $\begin{array}{l}\text { Hettich }{ }^{\circledR} \text { Universal } 320 \text { (Andreas } \\
\text { Hettich GmbH \& Co.KG, Tuttlingen, } \\
\text { Germany) }\end{array}$ \\
\hline Kumar et al. [43] & PRF & Not reported & 3000 rpm & 10 & Not reported \\
\hline Asmael et al. [58] & PRF & $\begin{array}{l}\text { Five or ten milliliters of intrave- } \\
\text { nous blood was drawn in } 10 \mathrm{~mL} \\
\text { glass vacuumed tube without } \\
\text { anticoagulants }\end{array}$ & 3000 rpm & 10 & $\begin{array}{l}\text { Centrifuge machine (Xiangtian, } \\
\text { Jiangsu China) }\end{array}$ \\
\hline Clark et al. [39] & A-PRF & $01 \mathrm{~mL}$ sterile glass vacuum tube & 1300 rpm & 8 & Not reported \\
\hline Alzahrani et al. [44] & PRF & Not reported & $3000(400 \times g)$ & 10 & $\begin{array}{l}\text { Compact centrifuge (Hermle } \\
\text { labortechnik, Germany) }\end{array}$ \\
\hline Temmerman et al. [50] & L-PRF & $\begin{array}{l}\text { Plastic } 10-m L \text { tubes without } \\
\text { anticoagulant }\end{array}$ & 2700 rpm & 12 & $\begin{array}{l}\text { (IntraSpin }{ }^{\mathrm{TM}} \text {, IntraLock, Boca Raton, } \\
\text { Florida, USA) }\end{array}$ \\
\hline Marenzi et al. [57] & L-PRF & 9-mL tubes & 2700 rpm & 12 & Intra-Lock, Boca-Raton, FL, USA \\
\hline Suttapreyasri et al. [45] & L-PRF & 10-mL glass tube & 3000 rpm & 10 & $\begin{array}{l}\text { Hettich Zentrifugen centrifuge } \\
\text { EBA } 20 \text { (Andreas Hettich GmbH\& } \\
\text { Co, KG, } \\
\text { Tuttlingen, Germany }\end{array}$ \\
\hline Hauser et al. [46] & PRF & $\begin{array}{l}\text { 8-mL tubes without anticoagu- } \\
\text { lant }\end{array}$ & 2700 rpm & 8 & Not reported \\
\hline Thakkar et al. [52] & PRF & $10 \mathrm{ml}$ syringe & 3000 rpm & 10 & Not reported \\
\hline Yewale et al. [51] & A-PRF + & $\begin{array}{l}\text { 10-mL tubes without antico- } \\
\text { agulants }\end{array}$ & 1300 rpm $(208 \times g)$ & 8 & Not reported \\
\hline
\end{tabular}

\section{Secondary outcomes}

The results of the secondary outcomes are described below.

\section{Pain assessment}

Six studies evaluated the patients pain reports using the visual analogue scale (VAS) by comparing socket treatment by means of PRF to spontaneous healing. $66.6 \%$ of the studies showed statistically significantly lower pain in the PRF group compared to the spontaneous wound 


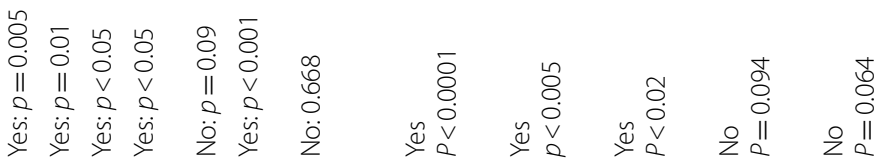

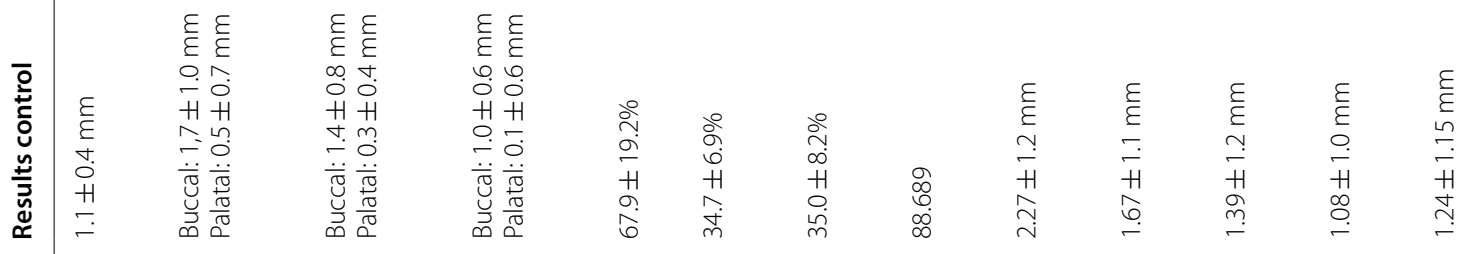

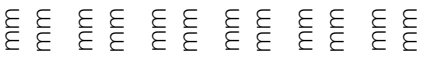

$\infty$

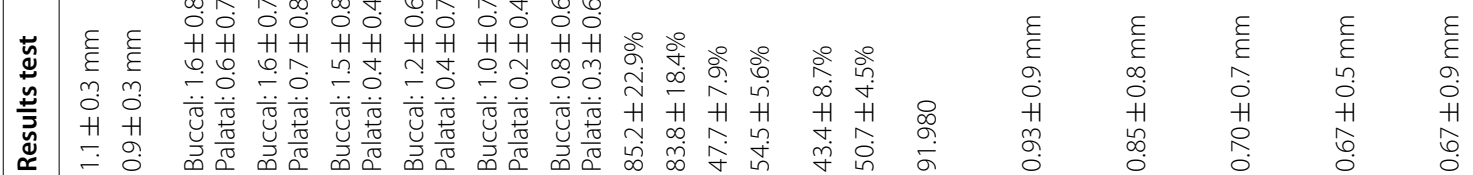




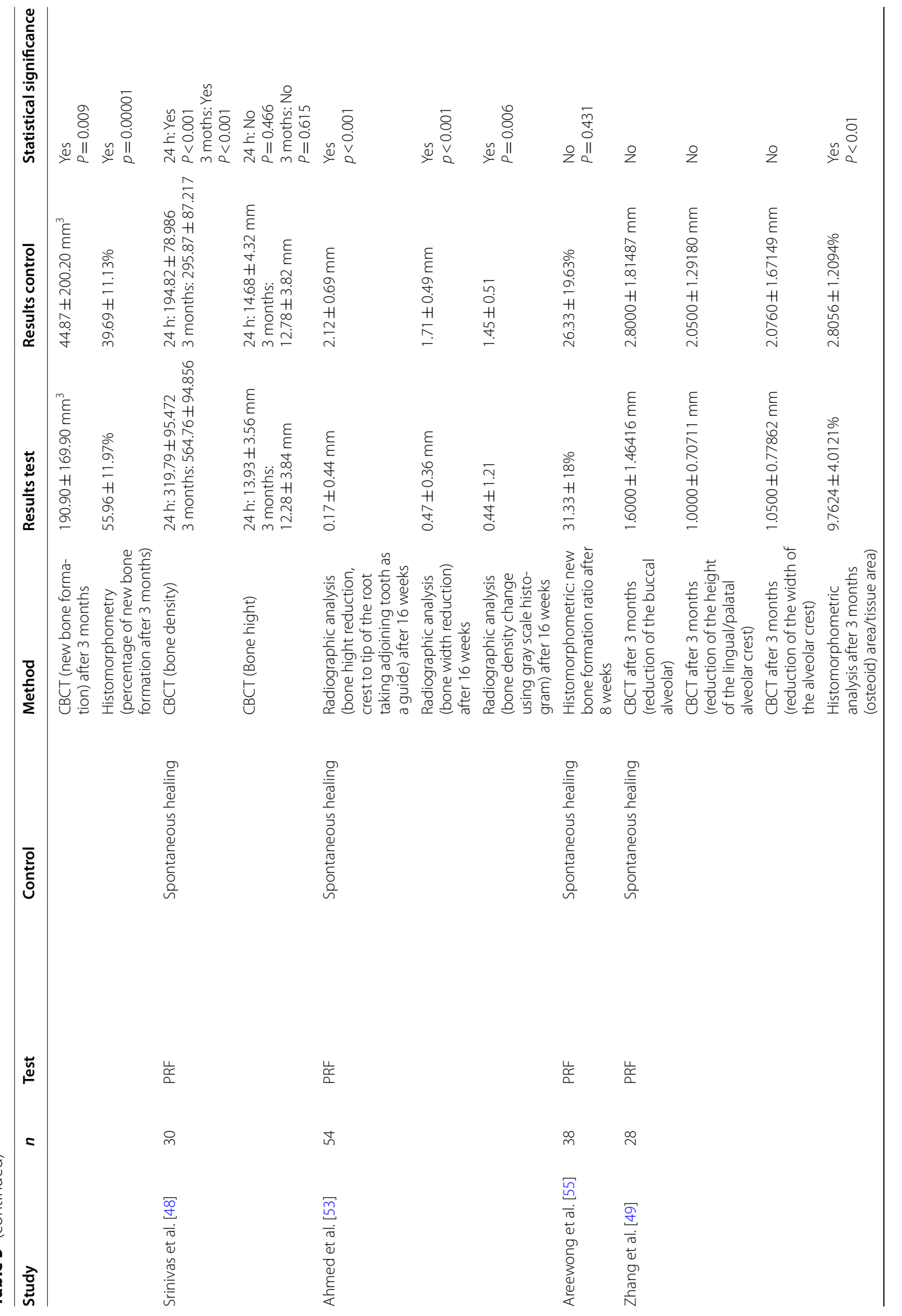




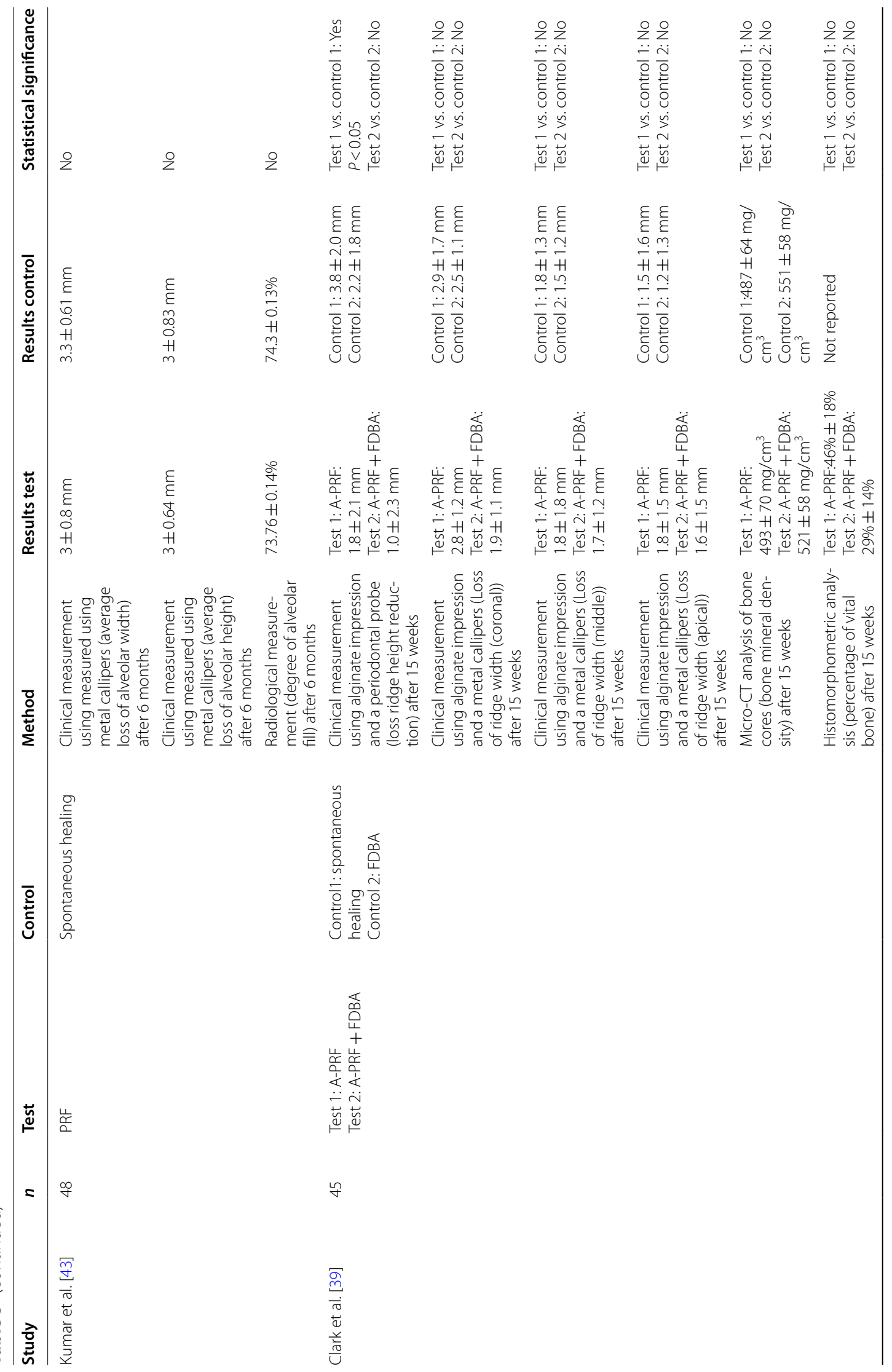




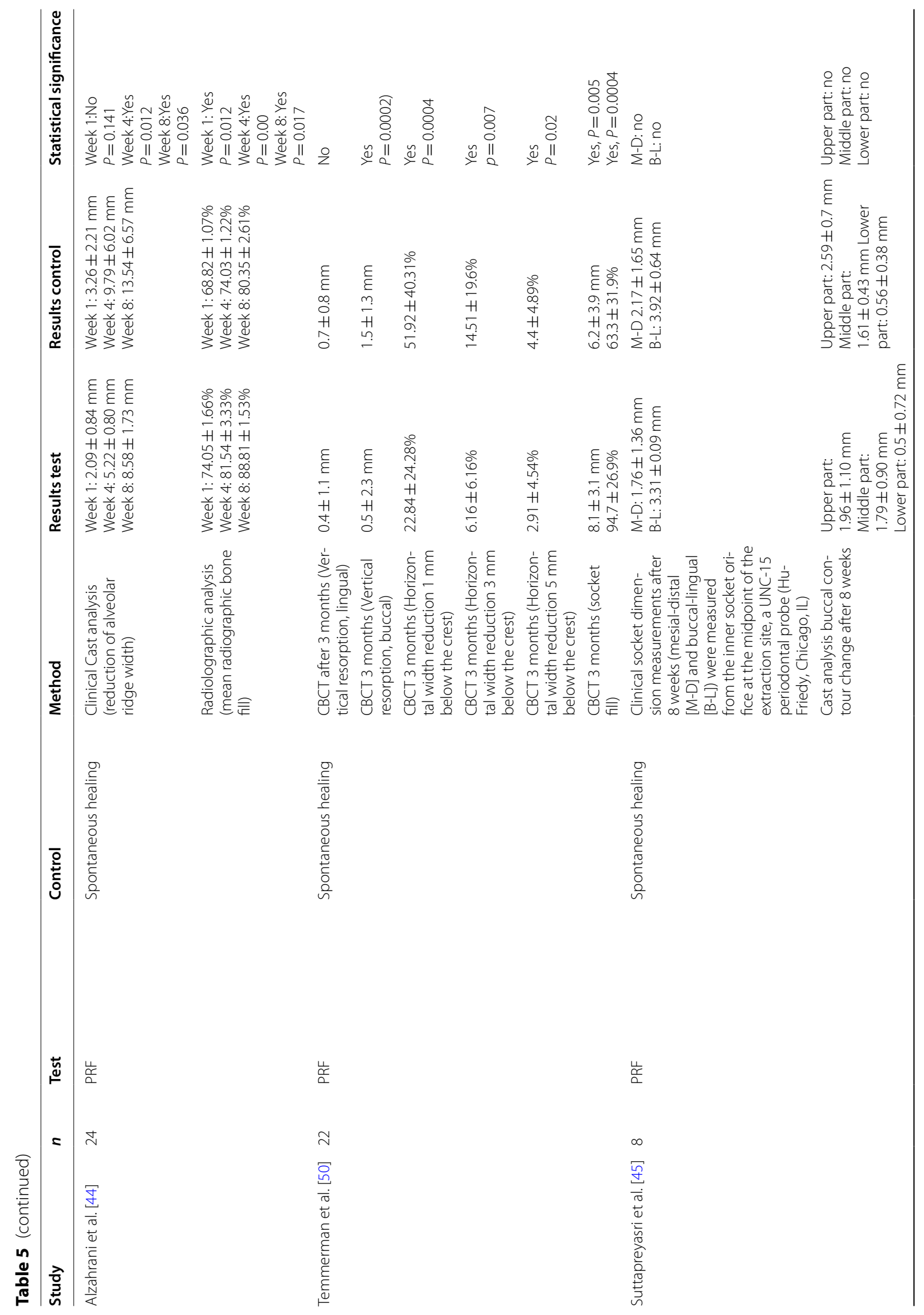




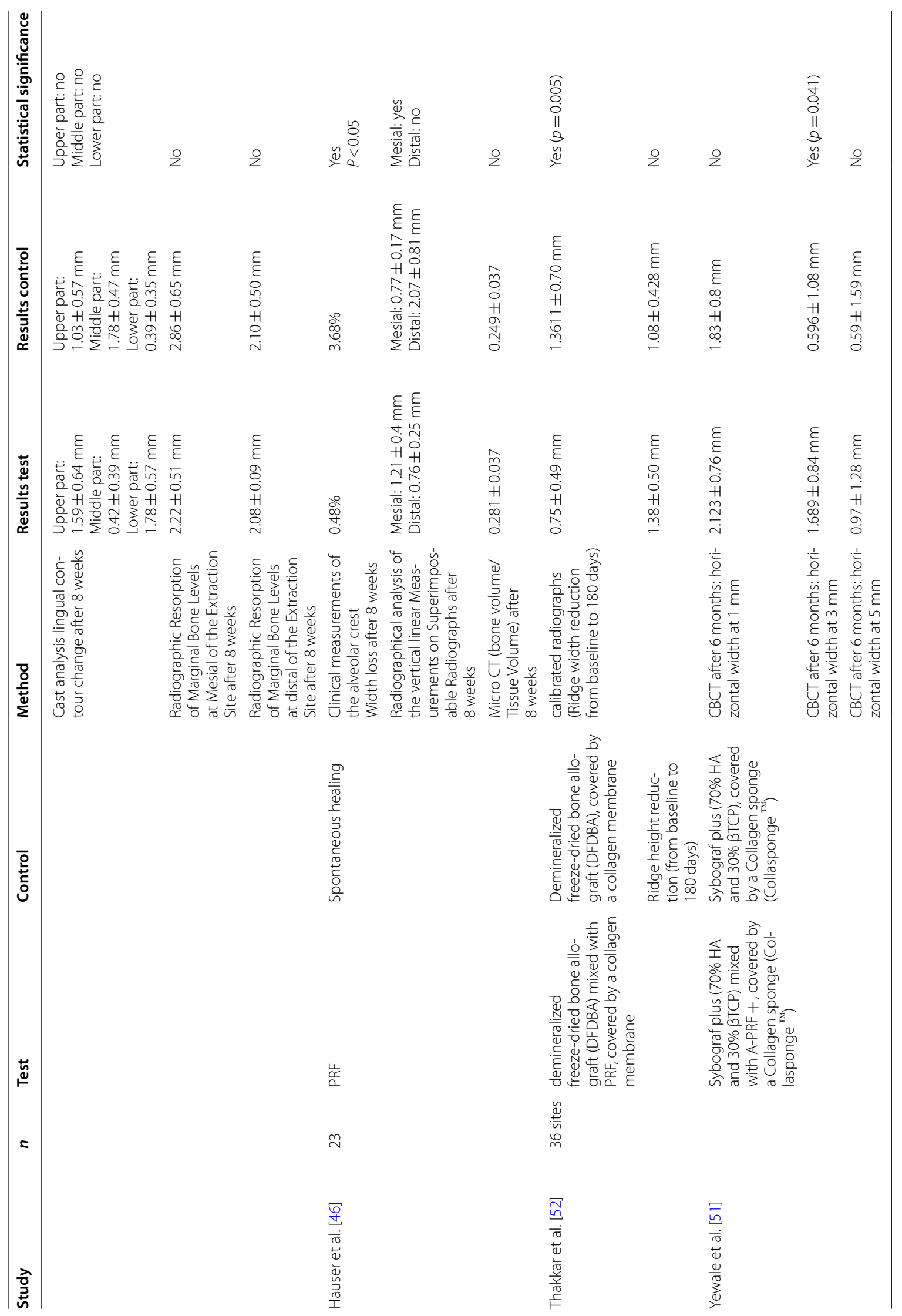


healing [42, 50, 56, 57]. Ustaoglu et al. [42] showed that both L-PRF and T-PRF significantly reduced patients' pain on day 1 compared to the control group of spontaneous wound healing. However, on day 2 the pain was reduced in both groups without statistical significant differences. Maurao et al. [56] showed that L-PRF significantly reduced patients pain on day 7 compared to the spontaneous healing without PRF. Kumar et al. [43] reported that $18 \%$ of the patients of the control group (spontaneous healing) reported on pain, whereas $0 \%$ of the PRF group had pain on day 1. Asmael et al. [58] did not show any statistical significant difference between the PRF treated side and the control side in their split mouth RCT. Tammerman et al. [50] evaluated patients pain on day 3 and showed that L-PRF significantly reduced the pain in comparison to the spontaneous wound healing. Additionally, Marenzi et al. [57] showed significant differences in the pain reduction of the L-PRF group compared to the spontaneous wound healing on early time point. However, the differences subsided on day 4, (Table 6).

One study by Yewale et al. [51] evaluated pain assessment after bone augmentation using bone substitute materials in combination with PRF versus bone substitute material alone ( $n=10$ per group). The results were not statistically significant.

\section{Soft tissue regeneration}

Soft tissue regeneration was evaluated in 8 studies, mainly using the soft tissue healing index by Landry et al.
[59]. Two studies showed no statistically significant differences between the L-PRF, A-PRF + groups compared to the spontaneous wound healing after one week [40, 42]. Six studies ( $75 \%$ of the evaluated studies) reported remarkable improvement of the soft tissue healing in the L-PRF and T-PRF groups compared to the spontaneous wound healing, especially in the early healing time point of one week [48, 53, 54, 56, 57]. Additionally, Ustaoglu et al. [42] evaluated the percent of epithelialization and showed statistically significantly faster epithelization in the L-PRF and T-PRF groups compared to the spontaneous wound healing on both time points week 1 and 2, whereas Asmael et al. [58] did not record any statistical significant difference between the evaluated groups (Table 7).

\section{Discussion}

Blood concentrates and especially PRF gained increasing interest in the oral and regenerative medicine in the last decade [31]. PRF is applied for different indications to support wound healing and regeneration of both bone and soft tissue. Recently, several systematic reviews evaluated the existing clinical evidence of PRF in different fields including oral and maxillofacial surgery $[33,60,61]$ and orthopedics [62]. However, most of recent reviews analyzed more than one indication and used a broad set of inclusion criteria, which hardly allow drawing concise conclusions for specific indications of PRF $[33,60,61]$. Additionally, focus was frequently placed on the general bone regeneration only, as an important parameter for

Table 6 Pain assessment outcomes in the evaluated studies

\begin{tabular}{|c|c|c|c|c|c|c|}
\hline Study & $n$ & Test & Control & Results test & Results control & Statistics \\
\hline Mourao et al. [56] & 32 & L-PRF & Spontaneous healing & 7 days: $4 \pm 1.15$ & 7 days: $5.12 \pm 1.08$ & Yes $(p=0.0128)$ \\
\hline \multirow[t]{3}{*}{ Ustaoglu et al. [42] } & \multirow[t]{3}{*}{57} & L-PRF & Spontaneous healing & $\begin{array}{l}\text { Day } 1: 3.30 \pm 2.07 \\
\text { Day 2: } 0.48 \pm 0.92\end{array}$ & \multirow[t]{3}{*}{$\begin{array}{l}\text { Day } 1: 5.11 \pm 1.60 \\
\text { Day } 2\end{array}$} & $\begin{array}{l}\text { Day 1: yes }(P=0.047) \\
\text { Day 2: No }\end{array}$ \\
\hline & & T-PRF & Spontaneous healing & $\begin{array}{l}\text { Day } 1: 3.29 \pm 1.85 \\
\text { Day 2: } 0.47 \pm 0.62\end{array}$ & & $\begin{array}{l}\text { Day 1: yes }(P=0.047) \\
\text { Day 2: No }\end{array}$ \\
\hline & & & & & & $\begin{array}{l}\text { T-PRF vs. L-PRF } \\
\text { No }\end{array}$ \\
\hline Kumar et al. [43] & 48 & PRF & Spontaneous healing & $\begin{array}{l}\text { Day 1: } 0 \% \text { of the } \\
\text { patients }\end{array}$ & $\begin{array}{l}\text { Day: } 118.1 \% \text { of the } \\
\text { patients }\end{array}$ & Not reported \\
\hline Asmael et al. [58] & 20 & PRF & Spontaneous healing & $\begin{array}{l}48 \mathrm{~h} \text { After Extraction: } \\
0.65\end{array}$ & $\begin{array}{l}48 \text { h After Extraction: } \\
1.8\end{array}$ & No \\
\hline Temmerman et al. [50] & 22 & L-PRF & Spontaneous healing & Day $3: 2,81$ & Day 3: 3,52 & $\begin{array}{l}\text { Yes } \\
P=0.03\end{array}$ \\
\hline Marenzi et al. [57] & 26 & L-PRF & Spontaneous healing & $3.2 \pm 0.3$ & $4.5 \pm 0.7$ & $\begin{array}{l}\text { Yes } \\
P<0.0001\end{array}$ \\
\hline Yewale et al. [51] & 20 & $\begin{array}{l}\text { Sybograf plus ( } 70 \% \text { HA } \\
\text { and } 30 \% \beta T C P) \text { mixed } \\
\text { with A-PRF +, covered } \\
\text { by a Collagen sponge } \\
\text { (Collasponge }^{\mathrm{TM}} \text { ) }\end{array}$ & $\begin{array}{l}\text { Sybograf plus ( } 70 \% \\
\text { HA and } 30 \% \beta \text { BCP), } \\
\text { covered by a Collagen } \\
\text { sponge (Collasponge } \\
\text { TM }\end{array}$ & $\begin{array}{l}\text { Pain frequency after } \\
10 \text { days: } \\
\text { Mild:2 } \\
\text { Moderate: } 8\end{array}$ & $\begin{array}{l}\text { Pain frequency after } \\
10 \text { days: } \\
\text { Mild:3 } \\
\text { Moderate: } 7\end{array}$ & No \\
\hline
\end{tabular}


Table 7 Soft tissue healing outcomes in the evaluated studies

\begin{tabular}{|c|c|c|c|c|c|c|c|}
\hline Study & $n$ & Test & Control & Method & Results test & Results control & Statistics \\
\hline Sharma et al. [54] & 30 & PRF & Spontaneous healing & $\begin{array}{l}\text { The Landry wound } \\
\text { healing index } \\
\text { (mean } \pm S D \text { ) }\end{array}$ & $\begin{array}{l}\text { Day } 3: 3.43 \pm 0.504 \\
\text { Day } 7: 3.93 \pm 0.254 \\
\text { Day } 14: 4.83 \pm 0.379\end{array}$ & $\begin{array}{l}\text { Day } 3: 3.17 \pm 0.379 \\
\text { Day } 7: 3.73 \pm 0.082 \\
\text { Day } 14: 4.3 \pm 0.46\end{array}$ & $\begin{array}{l}\text { Day 3: yes } \\
p=0.025 \\
\text { Day 7: yes } \\
P=0.039 \\
\text { Day 14: yes } \\
p=0.00\end{array}$ \\
\hline Mourao et al. [56] & 32 & L-PRF & Spontaneous healing & $\begin{array}{l}\text { Wound healing index } \\
\text { (mean } \pm S D)\end{array}$ & $\begin{array}{l}\text { Week 1: } 3.81 \pm 0.65 \\
\text { Week 2: } 4.75 \pm 0.44\end{array}$ & $\begin{array}{l}\text { Week 1: } 3.18 \pm 0.54 \\
\text { Week } 2: 4.5 \pm 0.51\end{array}$ & $\begin{array}{l}\text { Week 1: Yes } \\
p=0.0138 \\
\text { Week } 2: \text { No }\end{array}$ \\
\hline Srinivas et al. [48] & 30 & PRF & Spontaneous healing & $\begin{array}{l}\text { Wound healing index } \\
\text { after } 7 \text { days }\end{array}$ & $3.8 \pm 0.40$ & $3.0 \pm 0.53$ & $\begin{array}{l}\text { Yes } \\
P<0.001\end{array}$ \\
\hline Ahmed et al. [53] & & & Spontaneous healing & Wound healing index & Very good $94.1 \%$ & Very good in $86.7 \%$ & Not reported \\
\hline \multirow[t]{4}{*}{ Ustaoglu et al. [42] } & 57 & L-PRF & Spontaneous healing & $\begin{array}{l}\text { The Landry wound } \\
\text { healing index } \\
\text { (mean } \pm \text { SD) }\end{array}$ & $\begin{array}{l}\text { Week 1: } 3.58 \pm 0.63 \\
\text { Week 2: } 4.59 \pm 0.51\end{array}$ & $\begin{array}{l}\text { Week 1: } 3.21 \pm 0.66 \\
\text { Week 2: } 4.38 \pm 0.49\end{array}$ & $\begin{array}{l}\text { Week 1: no } \\
\text { Week 2: no }\end{array}$ \\
\hline & & & Spontaneous healing & $\begin{array}{l}\text { Complete wound } \\
\text { epithelization (\%) }\end{array}$ & $\begin{array}{l}\text { Week 1: } 54.9 \\
\text { Week 2: } 100\end{array}$ & $\begin{array}{l}\text { Week 1: } 10.1 \\
\text { Week 2: } 40.7\end{array}$ & $\begin{array}{l}\text { Week } 1: \text { yes } \\
P=0.047 \\
\text { Week } 2: \text { yes } \\
P=0.041\end{array}$ \\
\hline & & T-PRF & Spontaneous healing & $\begin{array}{l}\text { The Landry wound } \\
\text { healing index } \\
\text { (mean } \pm \text { SD) }\end{array}$ & $\begin{array}{l}\text { Week 1: } 3.69 \pm 0.51 \\
\text { Week 2: } 4.71 \pm 0.50\end{array}$ & $\begin{array}{l}\text { Week 1: } 3.21 \pm 0.66 \\
\text { Week 2: } 4.38 \pm 0.49\end{array}$ & $\begin{array}{l}\text { Week 1: no } \\
\text { Week 2: no }\end{array}$ \\
\hline & & & Spontaneous healing & $\begin{array}{l}\text { Complete wound } \\
\text { epithelization (\%) }\end{array}$ & $\begin{array}{l}\text { Week 1: } 70.1 \\
\text { Week 2: } 100\end{array}$ & $\begin{array}{l}\text { Week 1: } 10.1 \\
\text { Week 2: } 40.7\end{array}$ & $\begin{array}{l}\text { Week } 1 \text { : yes } \\
P=0.047 \\
\text { Week } 2 \text { : yes } \\
P=0.041\end{array}$ \\
\hline \multirow[t]{2}{*}{ Giudice et al. [40] } & 40 & $\mathrm{~A}-\mathrm{PRF}+$ & Spontaneous healing & $\begin{array}{l}\text { Wound healing index } \\
\text { (mean) }\end{array}$ & $\begin{array}{l}\text { Week 1: } 1 \\
\text { Week 2: } 0.25\end{array}$ & $\begin{array}{l}\text { Week 1: } 1.05 \\
\text { Week 2: } 0.33\end{array}$ & No \\
\hline & & L-PRF & Spontaneous healing & $\begin{array}{l}\text { Wound healing index } \\
\text { (mean) }\end{array}$ & $\begin{array}{l}\text { Week 1: } 0.95 \\
\text { Week 2: } 0.15\end{array}$ & $\begin{array}{l}\text { Week 1: } 1.05 \\
\text { Week 2: } 0.33\end{array}$ & No \\
\hline \multirow[t]{2}{*}{ Asmael et al. [58] } & 20 & PRF & Spontaneous healing & $\begin{array}{l}\text { Percentage of epitheli- } \\
\text { zation after } 1 \text { week }\end{array}$ & $52.7 \%$ & $51.3 \%$ & No \\
\hline & & & & $\begin{array}{l}\text { The Landry wound } \\
\text { healing index (mean) } \\
\text { after } 1 \text { week }\end{array}$ & 3.45 & 4.2 & $\begin{array}{l}\text { Yes } \\
P=0.0035\end{array}$ \\
\hline $\begin{array}{l}15 \\
\text { Marenzi et al. [57] }\end{array}$ & & L-PRF & Spontaneous healing & $\begin{array}{l}\text { Wound healing index } \\
\text { (mean } \pm S D)\end{array}$ & $\begin{array}{l}\text { Day } 3: 4.8 \pm 0.6 \\
\text { Day } 7: 4.5 \pm 0.5 \\
\text { Day } 14: 4.2 \pm 0.2 \\
\text { Day } 21: 4.1 \pm 0.1\end{array}$ & $\begin{array}{l}\text { Day } 3: 5.1 \pm 0.9 \\
\text { Day } 7: 4.9 \pm 0.3 \\
\text { Day 14: } 4.3 \pm 0.3 \\
\text { Day } 21: 4.2 \pm 0.2\end{array}$ & $\begin{array}{l}\text { Day 3: No } p=0.197 \\
\text { Day 7: yes } p=0.05 \\
\text { Day 14: yes } p=0.01 \\
\text { Day 21: yes } p=0.0002\end{array}$ \\
\hline
\end{tabular}

implantology, whereas little is known about the influence of PRF on specific parameters of wound healing including soft tissue regeneration and pain. Interestingly, these factors were shown to contribute to patients satisfaction and the long-term success of dental implants. Additionally, many studies did not use "reasonable" control groups thus involving several additional cofactors [61, 63]. For example several studies were conducted to compare PRF in the test group with a collagen-based biomaterials [64, $65]$ or mineralized bone substitute materials $[64,66]$ as a control group. In this context, it has to be noted that PRF is an autologous bioactive blood concentrate system based on the blood components including platelets and leukocytes, that are embedded in a fibrin network [21]. It does not exhibit the physicochemical characteristics of conventional biomaterials [21, 23]. Therefore, it is not comparable to other biomaterials such as bone substitute materials or collagen-based membranes. Accordingly, a precise control group is needed to evaluate the efficacy of PRF in the regeneration process. Hence, in this systematic review the native blood clot as a process of the spontaneous wound healing was considered as the most suitable and reasonable control group to assess the regenerative potential and efficacy of PRF. If biomaterials were utilized in combination with PRF, they had to be identical in the test and control groups. This restriction to reasonable control groups additionally aimed to exclude bias from additional cofactors potentially influencing the regeneration process. Based on this hypothesis, the present review addressed the following focused question: in 
patients with freshly extracted teeth, what is the efficacy of PRF in in the prevention of pain and the regeneration of soft tissue and bone compared to the respective control without PRF treatment?

The literature research revealed only 20 studies eligible for the evaluation. In total 17 studies (RCTs and CCTs) analyzed the effect of PRF compared to the spontaneous wound healing. One study included four groups and evaluated the treatment of PRF alone in the first test group compared to the spontaneous wound healing and in the second group the combination of PRF with a bone substitute material in the second test group compared to the bone substitute material alone in the second control group. Only 2 studies evaluated the combination of PRF with bone substitute materials in comparison to bone substitute material without PRF.

A relatively high bias risk was assessed for most of the studies, especially concerning blinding of patients and outcome assessment. Another limitation is the report on the morphology of the treated defects, i.e., the anatomy of the socket after tooth extraction in terms of the presence, quality and dimension of the buccal wall as well as the status of bone resorption at the time point of tooth extraction. Recent studies showed that among others these parameters are highly important for the progress of the regeneration process after tooth extraction and may predefine the risk of bone atrophy $[5,6]$. These limitations in the data acquisition point to the necessity to improve the quality of reporting in future studies.

Additionally, when evaluating PRF it is important to analyze the preparation protocol. PRF is not a ready-touse product, but a freshly prepared blood derivate for each individual patient. Recently, many different centrifugation protocols were reported in the literature [21, 29, $67,68]$. Additionally, there was a confusion in the literature concerning the reported parameters and the preparation methods $[69,70]$. Recent studies explored the role of the centrifugation process in the preparation of PRF [67, 71-77]. These studies have shown that the applied RCF has a crucial influence on the components and the bioactivity of PRF, thus influencing its therapeutic efficacy $[67,71-77]$. Thereby, the application of a high RCF during the centrifugation of PRF results in a significantly lower number of platelets, leukocytes and growth factor concentrations compared to PRF-matrices that are prepared using a low RCF [67, 71-77]. This phenomenon was proved in many studies and defined as the low-speed centrifugation concept (LSCC), which explained for the first time the role of the applied RCF in the preparation of blood concentrates [67]. In this context, three parameters are mainly important when reporting on the preparation of blood concentrates (a) the programmed revolutions per minutes $(\mathrm{rpm})$, which is a parameter that appears on the centrifuge in most types and is usually adjustable; (b) the applied centrifugal force (RCF), a parameter that is mostly not visible on the centrifuge but can be calculated according to the centrifuge radius and (c) the centrifugation time. Moreover, the used tube surface also influences the quality and bioactivity of the resulted PRF [78].

Most of the studies evaluated in the present review reported only the applied rpm, without any information about the radius of the used centrifuge or the resulted RCF. Fourteen of the studies referred to the first introduced protocol referred to "L-PRF" or "Choukrouns PRF" and used a relatively high rpm of 2700-3000 for 10 to $12 \mathrm{~min}$. Only three studies compared different PRF protocols including advanced PRF, that implements a medium RCF $(1300 \mathrm{rpm}, 208 \times g)$ or T-PRF, that implements specific titanium-based blood tubes. At this point, it has to be emphasized that the use of different preparation protocols results in different PRF-qualities that may manipulate the clinical outcome. Thereby, scientific reporting on PRF should include the above-mentioned parameters. Accordingly, the authors recommend a recently published guideline to report on the preparation of blood concentrates to be able to reproduce and evaluate the scientific data $[78,79]$.

Within the limitations of the acquired data, $66 \%$ of the evaluated studies showed that the application of PRF significantly reduced the postoperative pain, especially in early time points 1-3 days after surgery (Table 4). This observation may be explained by the autologous and bioactive character of PRF and the release of different growth factors and cytokines involved in pain control. The application of PRF provides the wound with all needed components to immediately start the healing process without the need for recruiting the immune cells to the injury area.

Additionally, $75 \%$ of the studies, that evaluated the influence of PRF on the soft tissue healing, showed that PRF promoted a significantly faster wound healing compared to the control group (Table 7). In this context, according to the wound healing index by Landry et al. [59], wound closure parameters were significantly better in the PRF group especially after 1 week of application. This finding reflects that PRF may be considered as an autologous wound healing booster to accelerate wound healing. Various studies have shown that PRF releases important growth factors such as epidermal growth factor (EGF), that promotes epithelialization, transforming growth factor beta (TGF- $\beta$ ), which is highly needed for fibroblasts proliferation and migration as well as vascular endothelial growth factor (VEGF), which is a key signal for neovascularization [67, 71-77].

The here reported clinical observations are in accordance with different preclinical studies showing the role 
of PRF in wound healing. In vitro studies used soft tissue regeneration model by combining fibroblasts and endothelial cells previously provided explanation on the possible mechanisms of PRF in promoting wound healing [74]. It was shown that in addition to the fibrin network, which provides a favorable scaffold for residual cells such as endothelial cells and fibroblasts, PRF serves as a drug delivery system by gradually releasing growth factors and promote the building of a well-defined vascular network as well as enhancing fibroblasts proliferation and migration [74, 77, 80]. Interestingly, the evaluated clinical studies reported mostly no significant difference in soft tissue healing after 2 weeks. This observation is logic, as the wound healing process under physiological conditions normally finalize after 2 weeks so that no differences between the evaluated groups are observed after this time period [81].

The analysis of the collected data concerning the efficacy of PRF in bone regeneration showed different outcomes according to the evaluation time point and applied method. Eleven studies reported on bone regeneration outcomes. Most of them evaluated bone regeneration after 8 to 15 weeks. Three of four studies reporting on clinical measurements showed significantly lower bone resorption in the PRF group compared to the control group, especially when considering the buccal wall and the ridge height. Similarly, CBCT evaluation of bone resorption, bone density and socket fill showed significantly lower resorption in the PRF group compared to the control group after 8-15 weeks. Especially, the 1-3 $\mathrm{mm}$ below the alveolar crest were well preserved in the PRF group compared to the control group. Interestingly, one study reported on bone regeneration after 6 months using clinical measurements and did not show any differences between the PRF and control group.

Within the limitations of these data, a very important finding may be highlighted by this analysis concerning the most suitable time point for implant insertion after socket preservation using PRF. Based on the present results, it seems that PRF promotes accelerated soft tissue and bone regeneration within the early healing phase. Apparently, PRF is effective in delaying bone resorption, but it cannot prevent it on the long run. Thereafter, the effect of PRF subsided, so that no difference could be observed after 6 months. These findings appear to be plausible when looking at the properties of PRF, which is an autologous, bioactive, fibrin-based scaffold, and different from ready-to-use biomaterials with stable scaffolds such as collagen-based biomaterials or bone substitute materials [67, 71-77]. An in vivo study has shown that PRF degrades after $2-3$ weeks, which is a sufficient time period to expand its effect on the early wound healing [23]. By contrast, the degradation time periods of conventional biomaterials such as collagen matrices or bone substitute materials ranged from 3 months to years according to the biomaterial specific characterization [16]. Therefore, when working with PRF, it is important to understand its characteristics as a fibrin-based scaffold and not as classical biomaterials.

Thereby, the present systematic review suggests considering PRF as a further group of regenerative biomaterials called blood-concentrates in addition to the xenogeneic, allogeneic and synthetic biomaterials. This specific group of blood concentrates provided completely different benefits and requirements and may be considered as an adjuvant therapy [82]. Accordingly, different treatment protocols apply for blood concentrates and they should not be treated likewise to the classical biomaterials in terms of guided bone regeneration (GBR) and guided tissue regeneration (GTR). Classical GBR/GTR biomaterials are inactive acellular materials, that require sufficient time until integrating into the implantation bed and allowing for cell migration and therefore initiation of the regeneration process $[83,84]$. Therefore, more time is needed in this case until the socket is ready for implantation. However, PRF is as a bioactive scaffold including crucial blood cells that are necessary for the regeneration process and can accelerate the phases of wound healing and starts the regeneration process earlier.

The physiological atrophy process after tooth extraction was described as a rapid and continuous process. About $50 \%$ of the alveolar bone atrophies in the first 3 months after tooth extraction $[7,85]$. Especially, in the first 3 months after tooth extraction the efficacy of PRF in delaying bone resorption was evidenced in the here evaluated studies. Consequently, after a period of 6 months the effect of PRF subsided and bone atrophy as described earlier. Only two studies were found, that evaluated the combination of bone substitute materials with PRF in comparison to the native bone substitute material without PRF. Based on the small number of patients and the limited data, it is not possible to draw a conclusion concerning the efficacy of PRF when combined with biomaterials. Therefore, further well-designed RCTs are needed to answer this question.

None of the here evaluated studies reported on the efficacy of PRF to reduce scar formation during soft tissue healing. Although liquid PRF is applied in esthetic treatment for skin rejuvenation and scar treatment $[86,87]$. Additionally, a recent study reported on the efficacy of PRF in promoting wound healing in large defects after three-dimensional augmentations in terms of the open healing concept as an alternative to flap mobilization and to avoid flap dehiscence [3]. Moreover, no data were found about the implant survival rate of implants placed in sockets treated with PRF compared to the spontaneous 
wound healing. Eventually, none of the evaluated studies reported on any adverse or server reactions related to the application of PRF.

Altogether, the analysis of the available evidence of 20 prospective, controlled studies highlighter the efficacy of PRF in supporting socket healing after tooth extraction. PRF was demonstrated to promote soft tissue regeneration, to reduce the postoperative pain and preventing bone dimensional bone loss in the early period of 2-3 months. This evidence refers to PRF protocols using a high RCF during the preparation. It has to be stated that the number of available studies in this field is very limited, and that the risk of bias was high. Future studies are needed to evaluate further PRF protocols using a lower RCF protocols to further investigate the potential benefit of different preparation protocols as an indication-specific approach.

\section{Conclusion}

The present reviews aimed to provide clinical evidence on the efficacy of PRF in the treatment of fresh extraction sockets in comparison to the spontaneous wound healing. Within the limitations of the collected data, PRF was found to be effective in reducing post-operative pain, accelerating soft tissue healing and preventing bone dimensional bone loss, especially in the early time period of 2-3 months. Although the present review focused only on prospective randomized controlled and controlled studies, a relatively high risk of bias was assessed, especially in the categories blinding of participant and personnel as well as blinding of outcome assessment. Additionally, the here evaluated data showed a high heterogeneity in the used methods for outcome measures. Therefore, it was not possible to perform a meta-analysis.

\begin{abstract}
Abbreviations
BSMs: Bone substitute materials; PRP: Platelet-rich plasma; PRGF: Plasma-rich in growth factors; PRF: Platelet-rich fibrin; L-PRF: Leukocytes-rich plateletrich fibrin; CCT: Controlled clinical trial; RCTs: Randomized controlled clinical trial; RPM: Round per minute; RCF: Relative centrifugal force; VAS: Visual analogue scale; CBCT: Cone beam computer tomography; Micro-C: Microcomputer tomography; GBR: Guided bone regeneration; GTR: Guided tissue regeneration.
\end{abstract}

\section{Acknowledgements}

Not applicable.

\section{Authors' contributions}

SA and SG performed the systematic research, data extraction and analysis and wrote the manuscript. KB and SA contributed to the methods and analysis of the extracted data. FS and RS were involved in the data management and interpretation. All authors read and approved the final manuscript.

\section{Funding}

Open Access funding enabled and organized by Projekt DEAL. This study was self-funded by the author.
Availability of data and materials

Not applicable.

\section{Declarations}

Ethical approval and consent to participate

Not applicable.

\section{Consent for publication \\ Not applicable.}

\section{Competing interests \\ The authors declare that they have no conflict of interests.}

\section{Author details}

${ }^{1}$ FORM, Frankfurt Oral Regenerative Medicine, Clinic for Maxillofacial and Plastic Surgery, Goethe University, Theodor-Stern-Kai 7, 60596 Frankfurt/ Main, Germany. ${ }^{2}$ Department of Oral Surgery and Implantology, Carolinum, Goethe University, Frankfurt, Germany. ${ }^{3}$ Department of Orthodontics, University of Düsseldorf, 40225 Düsseldorf, Germany.

Received: 12 August 2021 Accepted: 4 November 2021

Published online: 19 December 2021

\section{References}

1. Awadalkreem F, Khalifa N, Satti A, Suleiman AM. The influence of immediately loaded basal implant treatment on patient satisfaction. Int J Dent. 2020;2020:1.

2. Alrabiah M, Al Deeb M, Alsahhaf A, AlFawaz YF, Al-Aali KA, Vohra F, et al. Clinical and radiographic assessment of narrow-diameter and regulardiameter implants in the anterior and posterior jaw: 2 to 6 years of followup. J Periodontal Implant Sci. 2020;50(2):97.

3. Ghanaati S, Al-Maawi S, Conrad T, Lorenz J, Rössler R, Sader R. Biomaterialbased bone regeneration and soft tissue management of the individualized 3D-titanium mesh: An alternative concept to autologous transplantation and flap mobilization. J Cranio-Maxillofacial Surg. 2019 (cited 2019 Sep 5); https://linkinghub.elsevier.com/retrieve/pii/S1010518219304597.

4. Motamedian SR, Khojaste M, Khojasteh A. Success rate of implants placed in autogenous bone blocks versus allogenic bone blocks: a systematic literature review. Ann Maxillofac Surg. 2016;6(1):78-90.

5. Rothamel D, Schwarz F, Herten M, Engelhardt E, Donath K, Kuehn P, et al. Dimensional ridge alterations following socket preservation using a nanocrystalline hydroxyapatite paste. A histomorphometrical study in dogs. Int J Oral Maxillofac Surg. 2008;37(8):741-7.

6. Araújo MG, Silva CO, Misawa M, Sukekava F. Alveolar socket healing: what can we learn? Periodontol 2000. 2015;68(1):122-34.

7. Atwood DA. Reduction of residual ridges: a major oral disease entity. J Prosthet Dent. 1971;26(3):266-79.

8. Cawood Jl, Howell RA. A classification of the edentulous jaws. Int J Oral Maxillofac Surg. 1988;17(4):232-6.

9. McAllister BS, Haghighat K. Bone augmentation techniques. J Periodontol. 2007;78(3):377-96. https://doi.org/10.1902/jop.2007.060048.

10. Juodzbalys G, Stumbras A, Goyushov S, Duruel O, Tözüm TF. Morphological classification of extraction sockets and clinical decision tree for socket preservation/augmentation after tooth extraction: a systematic review. J oral Maxillofac Res. 2019;10(3):e3.

11. Al-Maawi S, Rutkowski J, Sader R, Kirkpatrick CJ, Ghanaati S. The biomaterial-induced cellular reaction allows a novel classification system regardless of the biomaterials origin. J Oral Implantol. 2020 (cited 2020 Mar 19); http://www.ncbi.n/m.nih.gov/pubmed/32068853.

12. Elgali I, Turri A, Xia W, Norlindh B, Johansson A, Dahlin C, et al. Guided bone regeneration using resorbable membrane and different bone substitutes: early histological and molecular events. Acta Biomater. 2016;29:409-23.

13. Tan Z, Kang J, Liu W, Wang H. The effect of the heights and thicknesses of the remaining root segments on buccal bone resorption in the socketshield technique: an experimental study in dogs. Clin Implant Dent Relat Res. 2018;20(3):352-9. 
14. Aghaloo TL, Moy PK. Which hard tissue augmentation techniques are the most successful in furnishing bony support for implant placement? Int J Oral Maxillofac Implants. 2007;22(Suppl):49-70.

15. Anwandter A, Bohmann S, Nally M, Castro AB, Quirynen M, Pinto N. Dimensional changes of the post extraction alveolar ridge, preserved with Leukocyte- and Platelet Rich Fibrin: a clinical pilot study. J Dent. 2016:52:23-9.

16. Al-Maawi S, Orlowska A, Sader R, James Kirkpatrick C, Ghanaati S. In vivo cellular reactions to different biomaterials—physiological and pathological aspects and their consequences. Semin Immunol. 2017;29:49-61.

17. Kattimani VS, Prathigudupu RS, Jairaj A, Khader MA, Rajeev K, Khader AA. Role of synthetic hydroxyapatite-in socket preservation: a systematic review and meta-analysis. J Contemp Dental Pract. 2019;20:987-93.

18. Esposito MAB, Koukoulopoulou A, Coulthard P, Worthington H V. Inter ventions for replacing missing teeth: dental implants in fresh extraction sockets (immediate, immediate-delayed and delayed implants). Cochrane Database Syst Rev. 2006

19. Lops D, Romeo E, Chiapasco M, Procopio RM, Oteri G. Behaviour of soft tissues healing around single bone-level-implants placed immediately after tooth extraction A 1 year prospective cohort study. Clin Oral Implants Res. 2013;24(11):1206-13.

20. Artzi Z, Nemcovsky CE, Dayan D. Bovine-HA spongiosa blocks and immediate implant placement in sinus augmentation procedures. Histopathological and histomorphometric observations on different histological stainings in 10 consecutive patients. Clin Oral Implants Res. 2002;13(4):420-7.

21. Ghanaati S, Booms P, Orlowska A, Kubesch A, Lorenz J, Rutkowski $J$, et al. Advanced platelet-rich fibrin: a new concept for cell-based tissue engineering by means of inflammatory cells. J Oral Implantol. 2014;40(6):679-89.

22. Schär MO, Diaz-Romero J, Kohl S, Zumstein MA, Nesic D. Platelet-rich concentrates differentially release growth factors and induce cell migration in vitro. Clin Orthop Relat Res. 2015:473(5):1635-43.

23. Kubesch A, Barbeck M, Al-Maawi S, Orlowska A, Booms PF, Sader RA, et al. A low-speed centrifugation concept leads to cell accumulation and vascularization of solid platelet-rich fibrin: an experimental study in vivo. Platelets. 2018; pp. 1-12.

24. Wend S, Kubesch A, Orlowska A, Al-Maawi S, Zender N, Dias A, et al. Reduction of the relative centrifugal force influences cell number and growth factor release within injectable PRF-based matrices. J Mater Sci Mater Med. 2017;28(12):188.

25. Kobayashi E, Flückiger L, Fujioka-Kobayashi M, Sawada K, Sculean A, Schaller B, et al. Comparative release of growth factors from PRP, PRF, and advanced-PRF. Clin Oral Investig. 2016; pp. 1-8.

26. Intini $\mathrm{G}$. The use of platelet-rich plasma in bone reconstruction therapy. Biomaterials. 2009:30:4956-66.

27. Anitua E, Sánchez M, Orive G, Andía I. The potential impact of the preparation rich in growth factors (PRGF) in different medical fields. Biomaterials. 2007;28(31):4551-60.

28. Anitua E, Andia I, Sanchez M. PRGF plasma rich growth factors. Dent dialogue. 2004; pp. 1-9.

29. Dohan DM, Choukroun J, Diss A, Dohan SL, Dohan AJJ, Mouhyi J, et al. Platelet-rich fibrin (PRF): A second-generation platelet concentrate. Part I: technological concepts and evolution. Oral Surg Oral Med Oral Pathol Oral Radiol Endodontol. 2006;101(3):e37.

30. Choukroun J, Diss A, Simonpieri A, Girard M-O, Schoeffler C, Dohan SL, et al. Platelet-rich fibrin (PRF): a second-generation platelet concentrate. Part IV: clinical effects on tissue healing. Oral Surg Oral Med Oral Pathol Oral Radiol Endodontol. 2006;101(3):56-60

31. Ghanaati S, Herrera-Vizcaino C, Al-Maawi S, Lorenz J, Miron RJ, Nelson K, et al. Fifteen years of platelet rich fibrin (PRF) in dentistry and oromaxillofacial surgery: how high is the level of scientific evidence? J Oral Implantol. 2018;44:471.

32. Öncü $E$, Alaaddinoğlu E. The effect of platelet-rich fibrin on implant stability. Int J Oral Maxillofac Implants. 2015;30(3):578-82.

33. Miron RJ, Zucchelli G, Pikos MA, Salama M, Lee S, Guillemette V, et al. Use of platelet-rich fibrin in regenerative dentistry: a systematic review. Clin Oral Investig. 2017;21(6):1913-27.

34. Miron RJ, Fujioka-Kobayashi M, Bishara M, Zhang Y, Hernandez M, Choukroun J. Platelet-rich fibrin and soft tissue wound healing: a systematic review. Tissue Eng Part B Rev. 2017;23(1):83-99.
35. Shamseer L, Moher D, Clarke M, Ghersi D, Liberati A, Petticrew M, et al. Preferred reporting items for systematic review and meta-analysis protocols (prisma-p) 2015: elaboration and explanation. BMJ. 2015;349:97647.

36. Page MJ, MCKenzie JE, Bossuyt PM, Boutron I, Hoffmann TC, Mulrow CD, et al. The PRISMA 2020 statement: an updated guideline for reporting systematic review. BMJ. 2021. https://doi.org/10.1136/bmj.n71.

37. Miller SA, Forrest JL. Enhancing your practice through evidence-based decision making: PICO, learning how to ask good questions. J Evid Based Dent Pract. 2001;1(2):136-41.

38. Higgins JPT, Thomas J, Chandler J, Cumpston M, Li T, Page MJ WV (editors). Cochrane Handbook for Systematic Reviews of Interventions version 6.2 (updated February 2021). Cochrane, 2021. www.training.cochr ane.org/handbook.

39. Clark D, Rajendran Y, Paydar S, Ho S, Cox D, Ryder M, et al. Advanced platelet-rich fibrin and freeze-dried bone allograft for ridge preservation: a randomized controlled clinical trial. J Periodontol. 2018:89(4):379-87.

40. Giudice A, Esposito M, Bennardo F, Brancaccio Y, Buti J, Fortunato L. Dental extractions for patients on oral antiplatelet: a within-person randomised controlled trial comparing haemostatic plugs, advancedplatelet-rich fibrin (A-PRF+) plugs, leukocyte- and platelet-rich fibrin (L-PRF) plugs and suturing alone; 2019.

41. Castro AB, Van Dessel J, Temmerman A, Jacobs R, Quirynen M. Effect of different platelet-rich fibrin matrices for ridge preservation in multiple tooth extractions: a split-mouth randomized controlled clinical trial. J Clin Periodontol. 2021:48:984

42. Ustaoğlu G, Göller Bulut D, Gümüş K. Evaluation of different platelet-rich concentrates effects on early soft tissue healing and socket preservation after tooth extraction. J Stomatol Oral Maxillofac Surg. 2019. https://doi. org/10.1016/j.jormas.2019.09.005.

43. Girish Kumar N, Chaudhary R, Kumar I, Arora SS, Kumar N, Singh H. To assess the efficacy of socket plug technique using platelet rich fibrin with or without the use of bone substitute in alveolar ridge preservation: a prospective randomised controlled study. Oral Maxillofac Surg. 2018;22(2):135-42.

44. Alzahrani AA, Murriky A, Shafik S. Influence of platelet rich fibrin on postextraction socket healing: a clinical and radiographic study. Saudi Dent J. 2017;29(4):149-55.

45. Suttapreyasri S, Leepong N. Influence of platelet-rich fibrin on alveolar ridge preservation. J Craniofac Surg. 2013;24(4):1088-94.

46. Hauser F, Gaydarov N, Badoud I, Vazquez L, Bernard JP, Ammann P. Clinical and histological evaluation of postextraction platelet-rich fibrin socket filling: a prospective randomized controlled study. Implant Dent. 2013;22(3):295-303.

47. dos Canellas JVS, da Costa RC, Breves RC, de Oliveira GP, da Figueredo CMS, Fischer RG, et al. Tomographic and histomorphometric evaluation of socket healing after tooth extraction using leukocyte- and platelet-rich fibrin: a randomized, single-blind, controlled clinical trial. J Cranio-Maxillofacial Surg. 2020;48(1):24-32.

48. Srinivas B, Das P, Rana MM, Qureshi AQ, Vaidya KC, Raziuddin SJA. Wound healing and bone regeneration in postextraction sockets with and without platelet-rich fibrin. Ann Maxillofac Surg. 2018;8(1):28-34.

49. Zhang Y, Ruan Z, Shen M, Tan L, Huang W, Wang L, et al. Clinical effect of platelet-rich fibrin on the preservation of the alveolar ridge following tooth extraction. Exp Ther Med. 2018;15(3):2277-86.

50. Temmerman A, Vandessel J, Castro A, Jacobs R, Teughels W, Pinto N, et al. The use of leucocyte and platelet-rich fibrin in socket management and ridge preservation: a split-mouth, randomized, controlled clinical trial. J Clin Periodontol. 2016:43(11):990-9.

51. Yewale M, Bhat S, Kamath A, Tamrakar A, Patil V, Algal AS. Advanced platelet-rich fibrin plus and osseous bone graft for socket preservation and ridge augmentation-a randomized control clinical trial. J Oral Biol Craniofacial Res. 2021;11(2):225-33.

52. Thakkar DJ, Deshpande NC, Dave DH, Narayankar SD. A comparative evaluation of extraction socket preservation with demineralized freezedried bone allograft alone and along with platelet-rich fibrin: a clinical and radiographic study. Contemp Clin Dent. 2016;7(3):371-6.

53. Ahmed N, Gopalakrishna V, Shetty A, Nagraj V, Imran M, Kumar P. Efficacy of PRF vs PRF + biodegradable collagen plug in post-extraction preservation of socket. J Contemp Dent Pract. 2019:20(11):1323-8.

54. Sharma A, Ingole S, Deshpande M, Ranadive P, Sharma S, Kazi N, et al. Influence of platelet-rich fibrin on wound healing and bone regeneration 
after tooth extraction: a clinical and radiographic study. J Oral Biol Craniofacial Res. 2020;10(4):385-90.

55. Areewong K, Chantaramungkorn M, Khongkhunthian P. Platelet-rich fibrin to preserve alveolar bone sockets following tooth extraction: a randomized controlled trial. Clin Implant Dent Relat Res. 2019;21(6):1156-63.

56. de Mourão ABCF, de Mello-Machado RC, Javid K, Moraschini V. The use of leukocyte- and platelet-rich fibrin in the management of soft tissue healing and pain in post-extraction sockets: a randomized clinical trial. J Cranio-Maxillofacial Surg. 2020:48(4):452-7.

57. Marenzi G, Riccitiello F, Tia M, di Lauro A, Sammartino G. Influence of leukocyte- and platelet-rich fibrin (L-PRF) in the healing of simple postextraction sockets: a split-mouth study. Biomed Res Int. 2015;205:369273.

58. Hm A, Fa J, Am H. Novel application of platelet-rich fibrin as a wound healing enhancement in extraction sockets of patients who smoke. J Craniofac Surg. 2018;29(8):E794-7.

59. Landry RTR, et al. Effectiveness of benzydamine $\mathrm{HCl}$ in the treatment of periodontal post-surgical patients. Res Clin Forums. 1988;10:105-18.

60. Dragonas P, Katsaros T, Avila-Ortiz G, Chambrone L, Schiavo JH, Palaiologou A. Effects of leukocyte-platelet-rich fibrin (L-PRF) in different intraoral bone grafting procedures: a systematic review. Int J Oral Maxillofacial Surg. 2019;48:250-62.

61. Strauss FJ, Stähli A, Gruber R. The use of platelet-rich fibrin to enhance the outcomes of implant therapy: a systematic review. Clin Oral Implants Res. 2018;29(Suppl 18):6-19.

62. Grecu AF, Reclaru L, Ardelean LC, Nica O, Ciucă EM, Ciurea ME. Plateletrich fibrin and its emerging therapeutic benefits for musculoskeletal injury treatment. Medicina (Kaunas). 2019;55(5):141.

63. Balli G, loannou A, Powell CA, Angelov N, Romanos GE, Soldatos N. Ridge preservation procedures after tooth extractions: a systematic review. Int J Dent. 2018;2018:1

64. Hartlev J, Schou S, Isidor F, Nørholt SE. A clinical and radiographic study of implants placed in autogenous bone grafts covered by either a platelet-rich fibrin membrane or deproteinised bovine bone mineral and a collagen membrane: a pilot randomised controlled clinical trial with a 2-year follow-up. Int J Implant Dent. 2021. https://doi.org/10.1186/ s40729-021-00289-z.

65. Hartlev J, Nørholt SE, Schou S, Isidor F. Pain after mandibular ramus block harvesting and lateral ridge augmentation with and without involvement of platelet-rich fibrin: a randomized controlled trial. Int J Oral Maxillofac Surg. 2021;50(3):384-90.

66. Olgun E, Ozkan SY, Atmaca HT, Yalim M, Hendek MK. Comparison of the clinical, radiographic, and histological effects of titanium-prepared platelet rich fibrin to allograft materials in sinus-lifting procedures. J Investig Clin Dent. 2018;9(4):e12347.

67. Choukroun J, Ghanaati S. Reduction of relative centrifugation force within injectable platelet-rich-fibrin (PRF) concentrates advances patients' own inflammatory cells, platelets and growth factors: the first introduction to the low speed centrifugation concept. Eur J Trauma Emerg Surg. 2018:44(1):87-95.

68. Fujioka-Kobayashi M, Schaller B, Mourão CFDAB, Zhang Y, Sculean A, Miron RJ. Biological characterization of an injectable platelet-rich fibrin mixture consisting of autologous albumin gel and liquid platelet-rich fibrin (Alb-PRF). Platelets. 2020;32:74.

69. Miron RJ, Choukroun J, Ghanaati S. Reply from authors: RE: optimized platelet-rich fibrin with the low-speed concept: growth factor release, biocompatibility, and cellular response: necessity for standardization of relative centrifugal force values in studies on platelet-rich fibrin. J Periodontol. 2019:90:122-5.

70. Miron R, Choukroun J, Ghanaati S. Controversies related to scientific report describing $\mathrm{g}$-forces from studies on platelet-rich fibrin: necessity for standardization of relative centrifugal force values. Int J Growth Factors Stem Cells Dent. 2018;1:80.

71. Wend S, Kubesch A, Orlowska A, Al-Maawi S, Zender N, Dias A, et al. Reduction of the relative centrifugal force influences cell number and growth factor release within injectable PRF-based matrices. J Mater Sci Mater Med. 2017. https://doi.org/10.1007/s10856-017-5992-6.

72. Kubesch A, Barbeck M, Al-Maawi S, Orlowska A, Booms PF, Sader RA, et al. A low-speed centrifugation concept leads to cell accumulation and vascularization of solid platelet-rich fibrin: an experimental study in vivo. Platelets. 2018;30:329
73. Ghanaati S, Al-Maawi S, Herrera-Vizcaino C, Alves GG, Calasans-Maia $M D$, Sader R, et al. A proof of the low speed centrifugation concept in rodents: new perspectives for in vivo research. Tissue Eng Part C Methods. 2018;24:659.

74. Herrera-Vizcaíno C, Dohle E, Al-Maawi S, Booms P, Sader R, Kirkpatrick CJ, et al. Platelet-rich fibrin secretome induces three dimensional angiogenic activation in vitro. Eur Cell Mater. 2019;37:250-64.

75. El Bagdadi K, Kubesch A, Yu X, Al-Maawi S, Orlowska A, Dias A, et al. Reduction of relative centrifugal forces increases growth factor release within solid platelet-rich-fibrin (PRF)-based matrices: a proof of concept of LSCC (low speed centrifugation concept). Eur J Trauma Emerg Surg. 2017;:45:467.

76. Al-Maawi S, Herrera-Vizcaino C, Dohle E, Zrnc TA, Parvini P, Schwarz F, et al, Homogeneous pressure influences the growth factor release profiles in solid platelet-rich fibrin matrices and enhances vascular endothelial growth factor release in the solid platelet-rich fibrin plugs. Int J Growth Factors Stem Cells Dent. 2018;1(1):8.

77. Dohle E, El Bagdadi K, Sader R, Choukroun J, James Kirkpatrick C, Ghanaat S. PRF-based matrices to improve angiogenesis in an in vitro co-culture model for bone tissue engineering. J Tissue Eng Regen Med. 2017. https://doi.org/10.1002/term.2475.

78. Ghanaati S, Mourão C, Adam E, Sader R, Zadeh H, Al-Maawi S. The role of centrifugation process in the preparation of therapeutic blood concentrates: standardization of the protocols to improve reproducibility. Int J Growth Factors Stem Cells Dent. 2019:2(3):41.

79. Miron RJ, Pinto NR, Quirynen M, Ghanaati S. Standardization of relative centrifugal forces in studies related to platelet-rich fibrin. J Periodontol. 2019;90:817-20.

80. Wang $X$, Yang $Y$, Zhang $Y$, Miron RJ. Fluid platelet-rich fibrin stimulates greater dermal skin fibroblast cell migration, proliferation, and collagen synthesis when compared to platelet-rich plasma. J Cosmet Dermatol. 2019;18(6):2004-10.

81. Gurtner $G$, Werner S, Barrandon Y, Longaker M. Wound repair and regeneration. Nature. 2008;453(7193):314-21. https://doi.org/10.1038/natur e07039.

82. Al-Maawi S, Herrera-Vizcaíno C, Orlowska A, Willershausen I, Sader R, Miron RJ, et al. Biologization of collagen-based biomaterials using liquidplatelet-rich fibrin: new insights into clinically applicable tissue engineering. Materials (Basel). 2019;12(23):3993.

83. Al-maawi S, Orlowska A, Sader R, Kirkpatrick CJ, Ghanaati S. In vivo cellular reactions to di ff erent biomaterials - physiological and pathological aspects and their consequences. Semin Immunol. 2017;29:49.

84. Ghanaati S, Al-Maawi S. Using bone repair materials in maxillofacial and skull surgery. In: Bone repair biomaterials. Elsevier; 2019. p. 361-78.

85. Atwood DA. Postextraction changes in the adult mandible as illustrated by microradiographs of midsagittal sections and serial cephalometric roentgenograms. J Prosthet Dent. 1963;13(5):810-24.

86. Ghanaati S, Al-Maawi S, Schaffner Y, Sader R, Choukroun J, Nacopoulos C. Application of liquid platelet-rich fibrin for treating hyaluronic acid-related complications: a case report with 2 years of follow-up. Int J Growth Factors Stem Cells Dent. 2018;1(2):74.

87. Nacopoulos C, Vesala AM. Lower facial regeneration with a combination of platelet-rich fibrin liquid matrices based on the low speed centrifugation concept-Cleopatra technique. J Cosmet Dermatol. 2020;19(1):185-9.

\section{Publisher's Note}

Springer Nature remains neutral with regard to jurisdictional claims in published maps and institutional affiliations. 